\title{
THE GAUSS MAP OF A GENUS THREE THETA DIVISOR
}

\author{
CLINT MCCRORY, THEODORE SHIFRIN AND ROBERT VARLEY
}

\begin{abstract}
A smooth complex curve is determined by the Gauss map of the theta divisor of the Jacobian variety of the curve. The Gauss map is invariant with respect to the $(-1)$-map of the Jacobian. We show that for a generic genus three curve the Gauss map is locally $Z / 2$-stable. One method of proof is to analyze the first-order $\mathbf{Z} / 2$-deformations of the Gauss map of a hyperelliptic theta divisor.
\end{abstract}

Andreotti's proof of the Torelli theorem shows that a smooth complex curve $C$ is determined by the Gauss map $\gamma$ of the theta divisor of the Jacobian variety of $C$. In fact, if $C$ is nonhyperelliptic, the branch locus of $\gamma$ is the dual hypersurface of the canonical embedding of $C$. But little is known about the relation between the intrinsic geometry of $C$ and the detailed structure of the singularities of $\gamma$. Of particular interest is how these singularities deform as the Jacobian varies among principally polarized abelian varieties. In this paper we show the importance of the symmetry of the Gauss map, by analyzing in detail the classical genus three case.

Let $x_{0}$ be a point of order two of the Jacobian variety $J(C)$. If $x_{0}$ lies on the theta divisor $\Theta$, then the germ of $\gamma$ at $x_{0}$ is not stable. In fact, $\gamma$ is invariant with respect to the involution induced on $\Theta$ by the map $x \rightarrow-x$ of $J(C)$, so $\gamma$ has rank zero at $x_{0}$. Since the singularity of $\gamma$ at $x_{0}$ has $\mathbf{Z} / 2$ symmetry, it is natural to ask whether $\gamma$ is stable as a $\mathbf{Z} / 2$-map. We prove that for the generic genus three curve, for all $x \in \Theta$, the germ of $\gamma$ at $x$ is a Z/2-stable holomorphic map germ:

Stability Theorem. For a smooth nonhyperelliptic curve $C$ of genus three, the Gauss map of the theta divisor is locally $\mathbf{Z} / 2$-stable if and only if all Weierstrass points of $C$ are normal.

It is surprising that a similar result holds even for hyperelliptic curves. The theta divisor $\Theta$ of the Jacobian of a hyperelliptic genus three curve has a singular point, and the Gauss map $\gamma$ on the smooth points of $\Theta$ extends to a map $\hat{\gamma}$ on the Nash blowup $\widehat{\Theta}$. The involution on $\Theta$ lifts to an involution on $\hat{\Theta}$ which now has the exceptional divisor as an extra component of its fixed point locus; $\hat{\gamma}$ is locally $\mathbf{Z} / 2$-stable for all smooth hyperelliptic genus three curves.

Received by the editors March 9, 1990. The results of this paper were presented by the third author at the meeting of the American Mathematical Society in Lawrence, Kansas, October 29, 1988.

1980 Mathematics Subject Classification (1985 Revision). Primary 14H40; Secondary 58C27.

Key words and phrases. Theta divisor, Jacobian variety, Gauss map, stable map germ, infinitesimal deformation, Kodaira-Spencer map. 
The $\mathbf{Z} / 2$-stable map germs of surfaces were classified by Bierstone [3]. If $\mathbf{Z} / 2$ acts trivially on the target, there are six types: the classical smooth, fold, and cusp germs of Whitney, together with three symmetric types. We find that all six of these types occur for Gauss maps of genus three theta divisors.

We begin in $\S 1$ with a discussion of $\mathbf{Z} / 2$-stability of holomorphic map germs. Using the theory of equivariant stability of maps, we show that a $Z / 2$-invariant map germ is $\mathbf{Z} / 2$-stable if and only if it is a $\mathbf{Z} / 2$-versal deformation of its fiber. We recall Bierstone's classification in dimension two, and we characterize $\mathbf{Z} / 2$ stable germs $\left(\mathbf{C}^{2}, 0\right) \rightarrow\left(\mathbf{C}^{2}, 0\right)$ by geometric properties of their singular loci (the transversality package).

In $\S 2$ we relate the singularities of the Gauss map to the extrinsic geometry of the canonical embedding of the nonhyperelliptic genus three curve $C$ as a plane quartic. By Riemann's theorem, $\Theta$ is identified with the symmetric square $C^{(2)}$ of $C$, and the Gauss map $\gamma: C^{(2)} \rightarrow \mathbf{P}^{2 *}$ is given by $\gamma(p+q)=\overline{p q}$, the secant line through $p$ and $q$. Thus the singularities of $\gamma$ reflect the contact of $C$ with lines in the plane. (Nonnormal Weierstrass points of $C$ correspond to higher flexes of the canonical curve.) We prove the stability theorem by checking the transversality package. The geometry of the canonical map is then used to show local $\mathbf{Z} / 2$-stability of $\hat{\gamma}$ in the hyperelliptic case. We briefly discuss a parallel deformation-theoretic proof of the stability theorem.

Since the generic three-dimensional principally polarized abelian variety is the Jacobian variety of a smooth genus three curve, a corresponding stability theorem holds for abelian varieties. In $\S 3$, we give a proof of the stability theorem using the first-order $\mathbf{Z} / 2$-symmetric deformations of the Gauss map of a hyperelliptic theta divisor. This variational proof shows how to analyze stability of Gauss maps for a family of hypersurfaces acquiring a double point. We include some background on Z/2-symmetric Kodaira-Spencer maps.

In dimension greater than three, the generic abelian variety is not a Jacobian. Using the dynamic method developed in $\S 3$, we plan to investigate the Gauss map of the theta divisor of the generic principally polarized abelian variety of dimension four. We expect that properties of the generic theta divisor can be seen from infinitesimal deformations of the theta divisor of a Jacobian. For such an analysis it is essential to use the Lagrangian structure of the Gauss map. In a sequel to this paper [17] we develop a theory of symmetric Lagrangian singularities appropriate to the study of Gauss maps of theta divisors.

The first author is grateful to the University of Warwick, and the second to Duke University, for support during the writing of this paper. The third author expresses his gratitude to the NSF for support under grant \#DMS-8803487. All three authors would like to thank Roy Smith for helpful and stimulating conversations.

\section{Z/2-STABILITY OF MAPS}

Mather's theory of stability of $C^{\infty}$ maps was extended to equivariant $C^{\infty}$ maps by Ronga, Poénaru, and Bierstone (cf. $[19,4,5]$ ). We describe the parallel theory of stability of equivariant holomorphic maps, specialized in three ways: (1) we assume the group $G$ of symmetries is finite; (2) we discuss stability of germs only; and (3) to define infinitesimal $V$-stability of a germ, we assume that $G$ acts trivially on the target. 
Let $G$ be a finite group acting holomorphically on the complex manifolds $X$ and $Y$. A holomorphic map $f: X \rightarrow Y$ is G-equivariant if $f(a x)=a f(x)$ for all $a \in G$. Two equivariant maps $f, g: X \rightarrow Y$ are $G$-equivalent if there exist equivariant analytic isomorphisms $h: X \rightarrow X$ and $k: Y \rightarrow Y$ such that $g=k \circ f \circ h^{-1}$. On the set of equivariant holomorphic maps from $X$ to $Y$, we consider the topology of uniform convergence on compact subsets. A $G$ equivariant holomorphic map $f: X \rightarrow Y$ is $G$-stable if there is a neighborhood $\mathscr{N}$ of $f$ in this topology such that if $g \in \mathcal{N}$ then $g$ is $G$-equivalent to $f$. We will be concerned only with the notion of local $G$-stability: Let $G_{x}=\{a \in$ $G: a x=x\}$. The holomorphic map $f: X \rightarrow Y$ is locally $G$-stable if the germ of $f$ at $x$ is $G_{x}$-stable for all $x \in X$.

The $G$-stability of a germ is defined as follows. Let $G$ be a finite group acting holomorphically on the complex manifold germs $\left(X, x_{0}\right)$ and $\left(Y, y_{0}\right)$. $(\operatorname{Re}-$ placing the action of $G$ by its derivative, one can assume $\left(X, x_{0}\right)=\left(\mathbf{C}^{m}, 0\right)$, $\left(Y, y_{0}\right)=\left(\mathbf{C}^{n}, 0\right)$, and $G$ acts linearly on $\mathbf{C}^{m}$ and $\mathbf{C}^{n}$.) A holomorphic map germ $f:\left(X, x_{0}\right) \rightarrow\left(Y, y_{0}\right)$ is $G$-equivariant if $f$ has a representative $\tilde{f}: U \rightarrow Y$ with $U$ open and $G U \subset U$, such that $\tilde{f}(a x)=a \tilde{f}(x)$ for all $a \in G$ and all $x \in U$. Two $G$-equivariant map-germs $f:\left(X, x_{0}\right) \rightarrow\left(Y, y_{0}\right)$ and $g:\left(X^{\prime}, x_{0}^{\prime}\right) \rightarrow\left(Y^{\prime}, y_{0}^{\prime}\right)$ are $G$-equivalent if there are equivariant analytic isomorphism germs $h:\left(X, x_{0}\right) \rightarrow\left(X^{\prime}, x_{0}^{\prime}\right)$ and $k:\left(Y, y_{0}\right) \rightarrow\left(Y^{\prime}, y_{0}^{\prime}\right)$ such that $g=k \circ f \circ h^{-1}$. A $G$-equivariant holomorphic map-germ $f:\left(X, x_{0}\right) \rightarrow\left(Y, y_{0}\right)$ is $G$-stable if it has a representative $\tilde{f}: U \rightarrow Y$ as above, with the following property. For every open set $U^{\prime} \subset U$ such that $G U^{\prime} \subset U^{\prime}$, there is a neighborhood $\mathscr{N}$ of $\tilde{f}$ in the space of $G$-equivariant holomorphic maps $U \rightarrow Y$ such that if $g \in \mathscr{N}$ there exists $x^{\prime} \in U^{\prime}$ with $G x^{\prime}=x^{\prime}$ and $f G$-equivalent to the germ of $g$ at $x^{\prime}$.

An equivariant map-germ $f$ is infinitesimally $G$-stable if every infinitesimal (first-order) equivariant perturbation of $f$ is the sum of infinitesimal equivariant coordinate changes in the source and target. More precisely, let $f:\left(X, x_{0}\right) \rightarrow\left(Y, y_{0}\right)$ be a holomorphic map-germ, equivariant with respect to the action of the finite group $G$ on the source and target. Then $f$ is infinitesimally $G$-stable if for every equivariant section germ $A$ of $f^{*} T Y$ at $x_{0}$, there exist equivariant section germs $H$ of $T X$ at $x_{0}$ and $K$ of $T Y$ at $y_{0}$ such that

$$
A(x)=d f_{x}(H(x))+K(f(x)) .
$$

(1.2) Theorem. Let $f$ be a G-equivariant holomorphic map-germ, where $G$ is a finite group acting holomorphically on the source and target of $f$. Then $f$ is $G$-stable if and only if $f$ is infinitesimally $G$-stable.

Proof. The real $C^{\infty}$ version of this result (for maps rather than germs) is due to Poénaru and Bierstone. A proof for $C^{\infty}$ germs has been given by Bierstone [5, Theorem 5.5, p. 121]. His proof adapts in a straightforward manner to holomorphic map-germs. The key ingredients in Bierstone's proof are equivariant versions of the preparation theorem and jet transversality. Both of these results are valid for equivariant holomorphic map-germs, at least when the group $G$ is finite. For the preparation theorem [5, Theorem 5.16], we replace Schwarz' theorem on invariant $C^{\infty}$ functions on a representation space of a compact Lie group [5, Theorem 2.6] with the corresponding result for invariant holo- 
morphic functions on a representation space of a finite group [6, Theorem 2]. To prove jet transversality, we need a holomorphic version of Bierstone's local transversality lemma [4, Lemma 9.3] $(r=1)$. Since his proof uses a polynomial perturbation, it is valid for analytic germs.

To define infinitesimal $G$ - $V$-stability of a germ $f:\left(X, x_{0}\right) \rightarrow\left(Y, y_{0}\right)$, we assume that $G$ acts trivially on $\left(Y, y_{0}\right)$. Thus $f$ is invariant if $f$ has a representative $\tilde{f}: U \rightarrow Y$ with $U$ open and $G U \subset U$, such that $\tilde{f}(a x)=\tilde{f}(x)$ for all $x \in U$. For simplicity (and to make an analogy with $[2, \S 6.6]$ ) we assume $\left(X, x_{0}\right)=\left(\mathbf{C}^{m}, 0\right),\left(Y, y_{0}\right)=\left(\mathbf{C}^{n}, 0\right)$, and $G$ acts linearly on $\mathbf{C}^{m}$. A map-germ $f:\left(\mathbf{C}^{m}, 0\right) \rightarrow\left(\mathbf{C}^{n}, 0\right)$ is invariant if and only if each coordinate $f_{i}$ is invariant. Let $\mathbf{A}_{x}=\mathscr{O}_{\mathbf{C}^{m}, 0}$ and $\mathbf{A}_{y}=\mathscr{O}_{\mathbf{C}^{n}, 0}$ denote the rings of germs of holomorphic functions on $\left(\mathbf{C}^{m}, 0\right)$ and $\left(\mathbf{C}^{n}, 0\right)$ respectively. Let $\mathbf{A}_{x}^{G}$ denote the ring of germs of $G$-invariant holomorphic functions $\left(\mathbf{C}^{m}, 0\right) \rightarrow \mathbf{C}$, and let $\left(\mathbf{A}_{x}^{n}\right)^{G}$ be the $\mathbf{A}_{x}^{G}$-module of germs of $G$-invariant holomorphic maps $\left(\mathbf{C}^{m}, 0\right) \rightarrow \mathbf{C}^{n}$. Then $\left(\mathbf{A}_{x}^{n}\right)^{G}=\left(\mathbf{A}_{x}^{G}\right)^{n}$, with $f$ corresponding to the $n$-tuple $\left(f_{1}, \ldots, f_{n}\right)$ of its coordinate functions. Let $x_{1}, \ldots, x_{m}$ and $y_{1}, \ldots, y_{n}$ be the standard coordinates on $\mathbf{C}^{m}$ and $\mathbf{C}^{n}$, respectively. We identify $\mathbf{A}_{x}^{n}$ with the module of germs of sections of $f^{*} T \mathbf{C}^{n}$, so that $\partial f / \partial x_{j} \in \mathbf{A}_{x}^{n}, j=1, \ldots, m$, and $e_{r}=\partial / \partial y_{r} \in \mathbf{A}_{x}^{n}, r=1, \ldots, n$. We define the tangent space, or firstorder $G$-deformation space, of $f$ to be the set of $G$-invariant elements of the quotient of $\mathbf{A}_{x}^{n}$ by the submodule generated by $\partial f / \partial x_{j}, j=1, \ldots, m$, and $f_{i} e_{r}, i, r=1, \ldots, n$ :

$$
T_{f}^{G}=\left(\mathbf{A}_{x}^{n} /\left\langle\partial f / \partial x_{j}, f_{i} e_{r}\right\rangle\right)^{G} .
$$

The motivation for this definition is given in [2, p. 129]. The module $\mathbf{A}_{x}^{n}$ is identified with germs of sections of $f^{*} T \mathbf{C}^{n}$, the space of all "variations" (or first-order perturbations) of $f$, and the submodule $\left\langle\partial f / \partial x_{j}, f_{i} e_{r}\right\rangle$ is the space of trivial variations (which do not change the isomorphism class of the variety $\left.f^{-1}(0)\right)$. By averaging, one proves

$$
T_{f}^{G}=\left(\mathbf{A}_{x}^{n}\right)^{G} /\left(\left\langle\partial f / \partial x_{j}, f_{i} e_{r}\right\rangle \cap\left(\mathbf{A}_{x}^{n}\right)^{G}\right),
$$

and $A \in\left\langle\partial f / \partial x_{j}, f_{i} e_{r}\right\rangle \cap\left(\mathbf{A}_{x}^{n}\right)^{G}$ if and only if

$$
A(x)=d f_{x}(H(x))+M(x) f(x),
$$

where $H:\left(\mathbf{C}^{m}, 0\right) \rightarrow\left(\mathbf{C}^{m}, 0\right)$ is an equivariant holomorphic germ and $M$ : $\left(\mathbf{C}^{m}, 0\right) \rightarrow\left\{\right.$ linear maps: $\left.\mathbf{C}^{n} \rightarrow \mathbf{C}^{n}\right\}$ is an invariant holomorphic germ. The germ $f$ is infinitesimally $G$ - $V$-stable if $T_{f}^{G}$ is generated as a complex vector space by $e_{1}, \ldots, e_{n}$. (In other words, every first-order perturbation of the variety of $f$ can be obtained from a trivial perturbation by means of an infinitesimal translation of the target space.)

(1.4) Theorem. Let $f$ be a G-invariant holomorphic map-germ, $G$ a finite group acting holomorphically on the source. Then $f$ is infinitesimally G-stable if and only if $f$ is infinitesimally $G$ - $V$-stable.

Proof (cf. [2, p. 129]). Comparing formulas (1.1) and (1.3), it is clear that infinitesimal $G$-stability of $f$ implies infinitesimal $G-V$-stability of $f$. Indeed, (1.1) says that $e_{1}, \ldots, e_{n}$ generate $\mathbf{M}=\left(\mathbf{A}_{x}^{n}\right)^{G} /\left(\left\langle\partial f / \partial x_{j}\right\rangle \cap\left(\mathbf{A}_{x}^{n}\right)^{G}\right)$ as an $\mathbf{A}_{y}^{G}$ module, and (1.3) says that the images of $e_{1}, \ldots, e_{n}$ span the complex vector 
space $\mathbf{M} / f^{*} \mathbf{m}_{y} \mathbf{M}$ (where $\mathbf{m}_{y}$ is the maximal ideal of $\mathbf{A}_{y}^{G}$ ). For the converse, we apply the holomorphic version of Bierstone's equivariant preparation theorem [5, Theorem 5.16, p. 130], which states that the homomorphism $f^{*}: \mathbf{A}_{y}^{G} \rightarrow \mathbf{A}_{x}^{G}$ is excellent. This means that if $\mathbf{M}$ is a finitely generated $\mathbf{A}_{x}^{G}$-module such that $\mathbf{M} / \mathbf{m}_{x} \mathbf{M}$ is a finite-dimensional vector space over $\mathbf{A}_{x}^{G} / \mathbf{m}_{x}$, then $M$ is finitely generated over $\mathbf{A}_{y}^{G}$. By Nakayama's lemma, if $\mathbf{M}$ is finitely generated over $\mathbf{A}_{y}^{G}$ and the images of the elements $e_{1}, \ldots, e_{n}$ of $\mathbf{M}$ span the vector space $\mathbf{M} / f^{*} \mathbf{m}_{y} \mathbf{M}$, then $e_{1}, \ldots, e_{n}$ generate $\mathbf{M}$ as an $\mathbf{A}_{y}^{G}$-module.

(1.5) Remark. It will be useful to characterize a locally $G$-stable map as a local $G$-versal deformation of its fibers (cf. [21, 8, p. 618]). If the $G$-invariant holomorphic germ $f:\left(X, x_{0}\right) \rightarrow\left(Y, y_{0}\right)$ is flat and $\left(X, x_{0}\right)$ and $\left(Y, y_{0}\right)$ are smooth, then $f$ is a deformation of the local analytic $G$-space $\left(F, x_{0}\right)=$ $\left(f^{-1}\left(y_{0}\right), x_{0}\right)$, and $T_{f}^{G}$ equals $\left(T_{\left(F, x_{0}\right)}^{1}\right)^{G}$, the space of first-order $G$-invariant deformations of $\left(F, x_{0}\right)$. Furthermore, $f$ is a $G$-versal deformation of $\left(F, x_{0}\right)$ if and only if the Kodaira-Spencer map $\varphi: T_{y_{0}}(Y) \rightarrow\left(T_{\left(F, x_{0}\right)}^{1}\right)^{G}$ is surjective [12]. The latter is precisely the condition that $f$ be infinitesimally $G$ - $V$-stable. (For $f:\left(\mathbf{C}^{m}, 0\right) \rightarrow\left(\mathbf{C}^{n}, 0\right)$ as above, $T_{y_{0}}(Y)=T_{0}\left(\mathbf{C}^{n}\right)$ is identified with $\mathbf{C}^{n}$, and $\varphi\left(e_{r}\right)$ is the image of $e_{r}$ in $T_{f}^{G}$.)

Now we focus on the special case of $\mathbf{Z} / 2$-invariant holomorphic germs $f$ : $\left(\mathbf{C}^{2}, 0\right) \rightarrow\left(\mathbf{C}^{2}, 0\right)$ and the classification of $\mathbf{Z} / 2$-stable singularities of such germs. The following theorem is a holomorphic version of Bierstone's classification [3] of $\mathbf{Z} / 2$-stable $C^{\infty}$ map-germs $\left(\mathbf{R}^{2}, 0\right) \rightarrow\left(\mathbf{R}^{2}, 0\right)$, specialized to the case when $\mathbf{Z} / 2$ acts trivially on the target. A holomorphic action of $Z / 2$ on the germ $\left(\mathbf{C}^{2}, 0\right)$ is equivalent to one of the three linear $\mathbf{Z} / 2$-spaces:

$$
\begin{aligned}
& T=\left(\mathbf{C}^{2}, 0\right) \text { with involution }(s, t) \rightarrow(s, t) \text { (the trivial involution), } \\
& A=\left(\mathbf{C}^{2}, 0\right) \text { with involution }(s, t) \rightarrow(-s,-t) \text { (the antipodal involution), } \\
& R=\left(\mathbf{C}^{2}, 0\right) \text { with involution }(s, t) \rightarrow(-s, t) \text { (reflection in the } t \text {-axis). }
\end{aligned}
$$

\begin{tabular}{|c|c|c|c|c|}
\hline $\begin{array}{c}\text { Symmetry } \\
\text { type }\end{array}$ & $\begin{array}{c}\text { Thom-Boardman } \\
\text { symbol }\end{array}$ & $\begin{array}{l}\text { Arnold-Giusti } \\
\text { symbol }\end{array}$ & Name & $\begin{array}{l}\text { Normal } \\
\text { form }\end{array}$ \\
\hline$T \rightarrow T$ & $\Sigma^{0}$ & & & $(s, t)$ \\
\hline$T \rightarrow T$ & $\bar{\Sigma}^{1,0}$ & $A_{1}$ & fold & $\left(s^{2}, t\right)$ \\
\hline$T \rightarrow T$ & $\Sigma^{1,1,0}$ & $A_{2}$ & cusp & $\left(s^{3}+s t, t\right)$ \\
\hline$A \rightarrow T$ & $\Sigma^{2,0}$ & $F^{2,2}$ & double fold & $\left(s^{2}, t^{2}\right)$ \\
\hline$R \rightarrow T$ & $\bar{\Sigma}^{1,0}$ & $A_{1}$ & symmetric fold & $\left(s^{2}, t\right)$ \\
\hline$R \rightarrow T$ & $\Sigma^{1,1,1,0}$ & $A_{3}$ & symmetric cusp & $\left(s^{4}+s^{2} t, t\right)$ \\
\hline
\end{tabular}

(1.6) Theorem. The $\mathbf{Z} / 2$-invariant holomorphic germ $f:\left(\mathbf{C}^{2}, 0\right) \rightarrow\left(\mathbf{C}^{2}, 0\right)$ is $\mathbf{Z} / 2$-stable if and only if it is equivalent to one of the following six germs:

Remarks. Note that the Thom-Boardman symbol and the symmetry type together characterize the equivalence class of a $\mathbf{Z} / 2$-stable invariant germ $\left(\mathbf{C}^{2}, 0\right)$ $\rightarrow\left(\mathbf{C}^{2}, 0\right)$. The Arnold-Giusti symbol is the singularity type of the variety $f^{-1}(0)$ at 0 (cf. [14, (7.19)]). When $\mathbf{Z} / 2$ is allowed to act nontrivially on the target, the holomorphic version of Bierstone's classification gives 16 types of Z/2-stable germs; cf. [3, pp. 524-525].)

Proof. Bierstone introduces certain jet transversality conditions which we call the transversality package for a germ $f$. He shows (1) the set of germs sat- 
isfying the transversality package is open and dense in the space of invariant germs, and (2) the transversality package implies that $f$ is equivalent to one of the above normal forms. His arguments work, with minor changes, for holomorphic germs. An easy computation shows that all of these normal forms are infinitesimally $\mathbf{Z} / 2-V$-stable, and hence $Z / 2$-stable by Theorems (1.2) and (1.4). Since the package is dense, it holds for any $Z / 2$-stable germ. Thus the transversality package, the normal forms, and $\mathbf{Z} / 2$-stability are equivalent.

The transversality package can be described in three equivalent ways: (a) jet transversality conditions, (b) explicit conditions on partial derivatives, and (c) geometric conditions on the singularity loci $\Sigma^{I}$. We now describe these three aspects of the package in detail. Conditions (a) and (b) are derived in [3], and (c) is an elementary computation in each case.

Let $f:\left(\mathbf{C}^{2}, 0\right) \rightarrow\left(\mathbf{C}^{2}, 0\right)$ be a $\mathbf{Z} / 2$-invariant holomorphic germ. Let $f^{k}$ denote the $k$-jet of $f$, and let $\Sigma_{k}^{I},|I| \leq k$, denote the subset of the $k$-jet space corresponding to the Thom-Boardman locus with symbol $\Sigma^{I}$. Let $\Sigma^{I}(f)=$ $\left(f^{k}\right)^{-1} \Sigma_{k}^{I}$, and $\bar{\Sigma}^{I}(f)=\operatorname{closure}\left(\Sigma^{I}(f)\right)$. We write $f(s, t)=(z, w)$, and if $0 \in \Sigma^{1}(f)$, we assume $w=t$. We also assume that the source of $f$ is one of the linear $\mathbf{Z} / 2$-spaces $T, A$, or $R$.

(1.7) The transversality package. (1) $\left(T \rightarrow T, \Sigma^{1,0}\right)$. With $f(s, t)=(z, t)$, we have $0 \in \Sigma^{1}(f) \Leftrightarrow z_{s}(0)=0$. The transversality conditions are: (a) $f^{1}$ is transverse to $\Sigma_{1}^{1},\left(\right.$ b) $z_{s s}(0) \neq 0$ or $z_{s t}(0) \neq 0$, (c) $\Sigma^{1}(f)$ is smooth at 0 , i.e., $\operatorname{grad}\left(\operatorname{det} d f_{x}\right)(0) \neq 0$.

(2) $\left(T \rightarrow T, \Sigma^{1,1}\right)$. With $f(s, t)=(z, t)$, we have $0 \in \Sigma^{1,1}(f) \Leftrightarrow z_{s}(0)=0$ and $z_{s s}(0)=0$. The transversality conditions are: (a) $f^{1}$ is transverse to $\Sigma_{1}^{1}$ and $f^{2}$ is transverse to $\Sigma_{2}^{1,1}$, (b) $z_{s t}(0) \neq 0$ and $z_{s s s}(0) \neq 0$, (c) $\Sigma^{1}(f)$ is smooth at 0 and $\operatorname{ker} d f_{x}$ has nondegenerate tangency with $\Sigma^{1}(f)$ at 0 ; i.e., the section of $\mathbf{P}\left(T \mathbf{C}^{2} \mid \Sigma^{1}(f)\right)$ defined by ker $d f_{x}$ is transverse to $\mathbf{P}\left(T \Sigma^{1}(f)\right)$ at 0 .

(3) $\left(T \rightarrow T, \Sigma^{2}\right)$. Let $f(s, t)=(z, w)$. Then $0 \in \Sigma^{2}(f) \Leftrightarrow z_{s}(0)=z_{t}(0)=$ $w_{s}(0)=w_{t}(0)=0$. The transversality condition is $\Sigma^{2}(f)=\varnothing$.

(4) $\left(A \rightarrow T, \Sigma^{2}\right)$. Let $f(s, t)=(z, w)$. Note that if $f$ has type $(A \rightarrow T)$, then $f$ has symbol $\Sigma^{2}$. Indeed, since $f(-s,-t)=f(s, t), z$ and $w$ are both even in $(s, t)$, so $0 \in \Sigma^{2}(f)$. The transversality conditions are: (a) the image of $\left(d f^{1}\right)_{0}$ is not tangent to the cone $\left\{z_{s} w_{t}-z_{t} w_{s}=0\right\}$, (b) the quadratic form $Q(\alpha, \beta)=\operatorname{det}\left(\left(d f^{1}\right)_{0}(\alpha, \beta)\right)$ is nondegenerate, (c) $\bar{\Sigma}^{1}(f)$ has an ordinary node at $0 \in \Sigma^{2,0}(f)$.

(5) $\left(R \rightarrow T, \Sigma^{1,0}\right)$. Note that if $f$ has type $(R \rightarrow T)$, then $f$ has symbol $\Sigma^{1}$. For with $f(s, t)=(z, t), z$ is even in $s$, so $z_{s}(0)=0$; i.e., $0 \in \Sigma^{1}(f)$. The transversality conditions are: (a) $f^{1}$ is transverse to $\Sigma_{1}^{1}$, (b) $z_{s s}(0) \neq 0$ or $z_{s t}(0) \neq 0,(\mathrm{c}) \Sigma^{1}(f)$ is smooth at 0 , i.e., $\operatorname{grad}\left(\operatorname{det} d f_{x}\right)(0) \neq 0$.

(6) $\left(R \rightarrow T, \Sigma^{1,1,1}\right)$. Note that if $f$ has type $(R \rightarrow T)$ and symbol $\Sigma^{1,1}$, then $f$ has symbol $\Sigma^{1,1,1}$. For with $f(s, t)=(z, t), z$ is even in $s$, so $z_{s s}(0)=0 \Rightarrow z_{s s s}(0)=0$. Thus $0 \in \Sigma^{1,1}(f) \Rightarrow 0 \in \Sigma^{1,1,1}(f)$. Let $D$ be the projection of Image $\left(d f^{3}\right)_{0}$ to the subspace $V$ of the 3-jet space spanned by $z_{s s}, z_{s t}$, and $z_{s s s}$. If $z_{s s}(0)=0$, then $D$ contains the line $z_{s t}=z_{s s s}=0$. The transversality conditions are: (a) $D$ is not tangent to the cone $\left\{z_{s s}=\right.$ $\left.0, z_{s t} z_{s s s}=0\right\},(\mathrm{b}) z_{s s t}(0) \neq 0$ and $z_{s s s s}(0) \neq 0$, (c) $\Sigma^{1}(f)$ has an ordinary 
node at $0 \in \Sigma^{1,1,1}(f)$, with one branch pointwise fixed by the involution, and ker $d f_{x}$ having nondegenerate tangency at 0 with the other branch.

(7) $\left(R \rightarrow T, \Sigma^{2}\right)$. The transversality condition is $\Sigma^{2}(f)=\varnothing$.

\section{Geometry of the Gauss maP}

Let $C$ be a smooth complex curve of genus three. The Jacobian variety $J(C)$ is a three-dimensional abelian variety; the theta divisor $\Theta$ of $J(C)$ is smooth if $C$ is not hyperelliptic. If $C$ is hyperelliptic, then $\Theta$ has a unique singular point $x_{0}$, which is an ordinary node. We define the Gauss map of $\Theta$ as follows. If $C$ is nonhyperelliptic, the Gauss map

$$
\gamma: \Theta \rightarrow \mathbf{P}\left(T_{0} J(C)\right)^{*}=\mathbf{P}^{2 *}
$$

assigns to $x \in \Theta$ the translate of the tangent plane $T_{x} \Theta$ to the origin of $J(C)$. If $C$ is hyperelliptic, let $\widehat{\Theta}$ be the Nash blowup of $\Theta$. The Gauss map

$$
\hat{\gamma}: \widehat{\Theta} \rightarrow \mathbf{P}\left(T_{0} J(C)\right)^{*}=\mathbf{P}^{2 *}
$$

is the unique extension of the tangent map $\Theta-\left\{x_{0}\right\} \rightarrow \mathbf{P}\left(T_{0} J(C)\right)^{*}$. The map $x \rightarrow-x$ of $J(C)$ takes the theta divisor $\Theta$ to itself, defining an involution $l: \Theta \rightarrow \Theta$. Thus $\Theta$ (resp. $\widehat{\Theta}$ in the hyperelliptic case) is a $Z / 2$-space, and the Gauss map is a $\mathbf{Z} / 2$-invariant map. We say that $\Theta$ is Gauss stable if the Gauss map $\gamma$ (resp. $\hat{\gamma}$ ) is a locally $\mathbf{Z} / 2$-stable map. Recall that $\gamma$ is locally $\mathbf{Z} / 2$-stable if, for every $x \in \boldsymbol{\Theta}$, the germ of $\gamma$ at $x$ is $(\mathbf{Z} / 2)_{x}$-stable. $\left((\mathbf{Z} / 2)_{x}=\mathbf{Z} / 2\right.$ if $x$ is a fixed point of $l$, and $(\mathbf{Z} / 2)_{x}=\{1\}$ if $x$ is not a fixed point of $l$.)

(2.1) Theorem. (a) Let $C$ be a smooth nonhyperelliptic curve of genus three. The theta divisor of its Jacobian variety is Gauss-stable if and only if all the Weierstrass points of $C$ are normal.

(b) If $C$ is any smooth hyperelliptic curve of genus three, then the theta divisor of its Jacobian variety is Gauss-stable.

(2.2) Corollary. The set of isomorphism classes of smooth nonhyperelliptic curves of genus three with Gauss-stable theta divisor is a nonempty Zariski-open subset of the moduli space $\mathscr{M}_{3}$ of smooth curves of genus three.

The corollary follows from the fact that the locus $W$ of isomorphism classes of curves with some nonnormal Weierstrass point is a divisor in $\mathscr{M}_{3}$.

Remark. The locus $W$ is the disjoint union of two divisors of $\mathscr{M}_{3}$ : (a) curves with some Weierstrass point of weight two but none of weight three, and (b) hyperelliptic curves, i.e., curves with all Weierstrass points of weight three. (Cf. [24].)

We prove (2.1) by using the synthetic geometry of the canonical model of $C$ to describe explicitly the singularities of the Gauss map. In (2.8) and (2.20), we check the geometric conditions on the singularity loci $\Sigma^{I}(\gamma)$ which comprise the transversality package (1.7). We also sketch an alternate proof of (2.1) by determining when the Gauss map is a local $\mathbf{Z} / 2$-versal deformation of its fibers (2.24).

Nonhyperelliptic curves. Let $C$ be a smooth nonhyperelliptic curve of genus three. Let $\left\{\omega_{1}, \omega_{2}, \omega_{3}\right\}$ be a basis for the holomorphic differentials on $C$. The canonical map

$$
\phi: C \rightarrow \mathbf{P}^{2}, \quad \phi(p)=\left(\omega_{1}(p), \omega_{2}(p), \omega_{3}(p)\right)
$$


gives an embedding of $C$ as a plane quartic curve. The Jacobian variety of $C$ is defined to be the torus

$$
J(C)=H^{0}\left(C, \Omega^{1}\right)^{*} / H_{1}(C, \mathbf{Z}) \cong \mathbf{C}^{3} / \Lambda,
$$

where $\Lambda$ is the period lattice of $C$ [9 and 1, Chapter 1]. The theta divisor $\Theta$ of $J(C)$ is, by Riemann's theorem, isomorphic to the symmetric square $C^{(2)}$ of $C$, the isomorphism being given by the Abel-Jacobi map

$$
\begin{gathered}
\mu: C^{(2)} \rightarrow J(C), \\
\mu(p+q)=\left(\int_{p_{0}}^{p} \omega_{j}+\int_{p_{0}}^{q} \omega_{j}\right)_{j=1,2,3} \bmod \Lambda .
\end{gathered}
$$

Identifying henceforth $\Theta$ with $C^{(2)}$, we see that the Gauss map of $\Theta$ is given by

$$
\begin{gathered}
\gamma: \Theta \rightarrow \mathbf{P}\left(T_{0} J(C)\right)^{*}=\mathbf{P}^{2 *}, \\
\gamma(p+q)=\mu_{*} T_{p+q} C^{(2)}, \\
\gamma(p+q)=[\phi(p) \wedge \phi(q)] \in \mathbf{P}\left(\Lambda^{2} \mathbf{C}^{3}\right)=\mathbf{P}^{2 *} .
\end{gathered}
$$

Identifying $C$ with its canonical model, we abbreviate this to

$$
\gamma(p+q)=p \wedge q .
$$

When $p=q$ the secant line is replaced as usual by the tangent line, viz., $\gamma(2 p)=\left[\phi(p) \wedge \phi^{\prime}(p)\right]=p \wedge p^{\prime}$. Note that the Gauss map has degree six: since $C$ has degree four, there are $\left(\begin{array}{l}4 \\ 2\end{array}\right)=6$ point pairs which map by $\gamma$ to a fixed line in $\mathbf{P}^{2}$. Abel's theorem implies that $l(p+q)=r+s$, where $p+q+r+s$ is a line section of the plane quartic $C$. (Note that $\gamma(p+q)=p \wedge q$ is patently $l$-invariant, since $[p \wedge q]=[r \wedge s]$ in this case.) Since the involution $l: \Theta \rightarrow \Theta$ is the restriction of the $(-1)$-map on $J(C), l$ is an isometry of $\Theta$ in its induced hermitian metric.

Let $\mathscr{U} \subset \mathscr{M}_{3}$ be the set of isomorphism classes of smooth nonhyperelliptic curves. Assigning to a curve its canonical model defines an isomorphism of $\mathscr{U}$ with the space of smooth plane quartics modulo projective equivalence. The Weierstrass points of a curve correspond to flex points of its canonical model $C$ $[9$, p. 274], and a Weierstrass point is normal if and only if the corresponding flex is ordinary, i.e., if the tangent line at the flex point has local intersection multiplicity three with $C$. A Weierstrass point is normal if and only if it has weight one, and the weight of a Weierstrass point is the local intersection multiplicity of $C$ and its hessian curve. Since the hessian of a quartic has degree six, the sum of the weights of the Weierstrass points is 24 .

Therefore Theorem (2.1a) is equivalent to the following

(2.7) Theorem. The theta divisor of the Jacobian variety of a smooth plane quartic $C$ is Gauss-stable if and only if $C$ has no higher flexes.

Remark. It can be verified directly that the set of curves with no higher flexes is a nonempty Zariski-open subset of the space of smooth plane quartics. For the condition that a quartic and its hessian intersect transversely is Zariski-open, and the Klein curve $\left\{x^{3} y+y^{3} z+z^{3} x=0\right\}$ has 24 distinct flexes (cf. [24, p. 12]). 
We prove Theorem (2.7) by using (2.6) to describe the geometry of the parabolic curve $\Pi=\overline{\Sigma^{1}}(\gamma)$, the singular locus $\Pi^{\prime}=\Sigma^{2}(\gamma)$, and the cusp locus $\mathfrak{C}=$ $\Sigma^{1,1}(\gamma)$ of the theta divisor $\Theta$.

(2.8) Theorem. Let $C$ be a smooth plane quartic.

(a) $\Pi=\left\{p+q \in C^{(2)}: p \neq q\right.$ and $\overline{p q}$ is tangent to $C$ at $p$ or $q$, or $p=q$ and $p$ is a flex point of $C\}$;

(b) $\mathfrak{C}=\{p+q \in \Pi: p$ is a flex point of $C\}$.

(c) $\Pi^{\prime}=\left\{p+q \in C^{(2)}: p \neq q\right.$ and $\overline{p q}$ is bitangent to $C$ at $p$ and $\left.q\right\}$;

(d) If $C$ has no higher flexes, then $\Pi$ is smooth except for ordinary nodes at $\Pi^{\prime}$, and $\operatorname{ker} d \gamma$ has nondegenerate tangency with $\Pi$ at $\mathfrak{C}$.

(e) If $C$ has a higher flex at $p$, then $2 p=p+p$ is a tacnode of $\Pi$.

Remark. A plane quartic has 28 bitangents and 24 flexes, so $\Pi^{\prime}$ consists of 28 points and $\mathfrak{C}$ of 48 points, provided that $C$ has no higher flexes.

Theorem (2.8) implies (2.7) as follows. The intersection of a line with the plane quartic $C$ has one of the following forms:

(i) $p+q+r+s$, with $p, q, r, s$ distinct,

(ii) $2 p+q+r$, with $p, q, r$ distinct,

(iii) $2 p+2 q$, with $p, q$ distinct,

(iv) $3 p+q$, with $p, q$ distinct,

(v) $4 p$.

Thus $(2.8 \mathrm{a}, \mathrm{b}, \mathrm{c})$ says that if $C$ has no higher flexes, then the only singularity types of $\gamma$ which occur are $\left(T \rightarrow T, \Sigma^{1,0}\right)(2.8 \mathrm{a}),\left(T \rightarrow T, \Sigma^{1,1}\right)(2.8 \mathrm{~b})$, and $\left(A \rightarrow T, \Sigma^{2}\right)(2.8 \mathrm{c})$. Condition (2.8d) says that parts (1), (2), (4) of the transversality package (1.7) hold. Since no other singularity types of $\gamma$ occur, parts (3), (5), (6), (7) of (1.7) hold. On the other hand, if $C$ has a higher flex, then (2.8e) implies that (1.7) does not hold.

We turn now to the proof of (2.8). We verify (2.8) first away from the diagonal $\Delta=\{p+p\} \subset C^{(2)}$, and then we check it for this case.

We begin by calculating the derivative of the Gauss map at the point $p+q \in$ $C^{(2)}, p \neq q$. If $s$ and $t$ are local coordinates on $C$ near $p, q$ respectively, then differentiating (2.6) yields

$$
d \gamma=p^{\prime} \wedge q d s+p \wedge q^{\prime} d t \quad \bmod (p \wedge q) .
$$

It will be useful to have a formula for the second fundamental form II of $\Theta$. Recall that if $\gamma: \Theta \rightarrow \mathbf{P}^{2 *}$, then $d \gamma: T \Theta \rightarrow T \mathbf{P}^{2 *} \cong \operatorname{Hom}(E, Q)$, where $E$ is the tautological rank 2 (sub)bundle on $\mathbf{P}^{2 *}$ and $Q$ is the rank 1 quotient bundle. Since $\gamma^{*} E \cong T \Theta$, the fundamental result is that

$$
d \gamma: T \Theta \rightarrow \operatorname{Hom}\left(T \Theta, \mathbf{C}^{3} / T \Theta\right)=\operatorname{Hom}\left(T \Theta, N_{\boldsymbol{\Theta}}\right)
$$

is a symmetric bundle map (cf. $[15,16])$. The associated quadratic form II: $\operatorname{Sym}^{2}(T \Theta) \rightarrow N_{\Theta}$ is called the second fundamental form of $\Theta$.

To use (2.9) to describe II, we need to understand the tangent isomorphism induced by the isomorphism $\mathbf{P}\left(\Lambda^{2} \mathbf{C}^{3}\right) \cong \mathbf{P}^{2 *}$. Let $\mathscr{L}$ be the tautological line bundle on $\mathbf{P}\left(\Lambda^{2} \mathbf{C}^{3}\right)$. Then $T \mathbf{P}\left(\Lambda^{2} \mathbf{C}^{3}\right) \cong \operatorname{Hom}\left(\mathscr{L}, \Lambda^{2} \mathbf{C}^{3} / \mathscr{L}\right)$, and so the isomorphism $T \mathbf{P}\left(\Lambda^{2} \mathbf{C}^{3}\right) \cong T \mathbf{P}^{2 *}$ is given by a pairing

$$
\operatorname{Hom}\left(\mathscr{L}, \Lambda^{2} \mathbf{C}^{3} / \mathscr{L}\right) \otimes E \rightarrow Q .
$$


Tensoring with $\mathscr{L}$, we have

$$
\Lambda^{2} \mathbf{C}^{3} / \mathscr{L} \otimes E \rightarrow Q \otimes \mathscr{L} .
$$

Since $\mathscr{L} \cong \Lambda^{2} E$, it follows that $Q \otimes \mathscr{L} \cong \Lambda^{3} \mathbf{C}^{3}$, and the pairing is given by $\phi \otimes v \mapsto \phi \wedge v$.

(2.10) Proposition. At a point $p+q \in C^{(2)}-\Delta$, with the local coordinates $s, t$ as above, the second fundamental form II is given by the matrix

$$
\mathbf{I I}=\left[\begin{array}{cc}
p \wedge q \wedge p^{\prime} & 0 \\
0 & p \wedge q \wedge q^{\prime}
\end{array}\right] .
$$

Proof. From (2.4) it follows that $T_{p+q} \Theta \cong \operatorname{Span}(p, q) \subset T_{0} J(C)$; the tangent vectors $\partial / \partial s$ and $\partial / \partial t$ correspond to $p$ and $q$ respectively. Let $\xi \in \mathbf{P}\left(\Lambda^{2} \mathbf{C}^{3}\right)$ be spanned by $p \wedge q$, and let $\phi=d \gamma(\partial / \partial s)$. Then $\mathbf{I I}(\partial / \partial s, \partial / \partial s)=\phi \wedge p=$ $p^{\prime} \wedge q \wedge p$, and $\mathbf{I I}(\partial / \partial s, \partial / \partial t)=p^{\prime} \wedge q \wedge q=0$. Similarly, $\mathbf{I I}(\partial / \partial t, \partial / \partial t)=$ $p \wedge q^{\prime} \wedge q$. By choosing an appropriate basis for $\Lambda^{3} C^{3}$, we may write the matrix as given.

We see from (2.10), or directly from (2.9), that for $p \neq q$, the parabolic curve $\Pi$ is given by

$$
\Pi=\left\{p+q \in C^{(2)}: p \wedge p^{\prime} \wedge q=0\right\},
$$

which implies $(2.8 \mathrm{a})$ for $p \neq q$. Moreover, $\gamma$ has rank zero at a point $p+q$ such that $p \wedge p^{\prime} \wedge q=p \wedge q \wedge q^{\prime}=0$; in this case, the line $\overline{p q}$ is bitangent to $C$ at $p$ and $q$, which proves $(2.8 \mathrm{c})$ for $p \neq q$.

The cusp locus $\mathfrak{C}=\Sigma^{1,1}(\gamma)$ is described as follows. The point $P=p+q$ is an element of $\mathfrak{C}$ if and only if the following Lagrange multipliers condition holds:

$$
\begin{aligned}
d \gamma & \equiv 0 \bmod \left(\gamma, d\left(p \wedge p^{\prime} \wedge q\right)\right) \quad \text { on } \Pi \\
& \Leftrightarrow p \wedge q^{\prime} d t \equiv 0 \bmod \left(p \wedge q, p \wedge p^{\prime \prime} \wedge q d s+p \wedge p^{\prime} \wedge q^{\prime} d t\right) \quad \text { on } \Pi .
\end{aligned}
$$

(For example, if $x$ is a smooth point of $\Pi$, then $x \in \mathfrak{C}$ if and orily if $d(\gamma \mid \Pi)_{x}=$ 0 , i.e., $\operatorname{ker}(d \gamma)_{x}=T_{x} \Pi$.) There are two possibilities: if $p \wedge q^{\prime}=0 \bmod (p \wedge q)$, then $p+q$ is a point of $\Pi^{\prime}$ and is therefore ruled out; if $p \wedge q^{\prime} \neq 0 \bmod (p \wedge q)$, then the condition is equivalent to $p \wedge p^{\prime \prime} \wedge q=0$, i.e., $p$ is a flex point of $C$. This proves $(2.8 \mathrm{~b})$ for $p \neq q$.

If the line $l=\overline{p q}$ is simply tangent to $C$ at $p$ and not tangent to $C$ at $q$, we may take local parametrizations $p(s)=\left(1, s, s^{2}+\cdots\right)$ and $q(t)=(\alpha t+$ $\cdots, 1, t)$; then $\gamma(s, t)=\left(s t-s^{2}+\cdots,-t+\cdots, 1\right)$, and $\Pi$ is smooth at $p+q$. If $l=\overline{p q}$ is bitangent to $C$ at $p$ and $q$, then we may take $p(s)=\left(1, s, s^{2}+\cdots\right)$ and $q(t)=\left(t, 1, t^{2}+\cdots\right)$, and $\gamma(s, t)=\left(-s^{2}+\cdots,-t^{2}+\cdots, 1\right)$ and so $\Pi$ has an ordinary node at $p+q$. If $l$ is doubly tangent to $C$ at $p$ (so that $p$ is a flex of $C)$, but not tangent to $C$ at $q$, then we use $p(s)=\left(1, s, s^{3}+\cdots\right), q(t)=$ $\left(\alpha t^{2}+\cdots, 1, t\right)$, and so $\gamma(s, t)=\left(s t-s^{3}+\cdots,-t+\cdots, 1\right)$, and once again $\Pi$ is smooth at $p+q$. In fact, $\Pi=\left\{t-3 s^{2}+\cdots=0\right\}$, and (2.9) implies that $\operatorname{ker} d \gamma$ is spanned by $\partial / \partial s$ for all $p+q \in \Pi$, and so we see that $\operatorname{ker} d \gamma$ has nondegenerate tangency with $\Pi$ for $p \neq q$. Since (2.8e) is vacuous for $p \neq q$, we have verified $(2.8)$ for $p \neq q$.

We have so far ignored the diagonal $\Delta \subset C^{(2)}$. To come to grips with the behavior of $\gamma$ at a point $2 p=p+p$, let $s, t$ be local coordinates on $C$ centered 
at $p$, and let $u=s+t, v=s t$ be the elementary symmetric functions. Now, in terms of the coordinates $u, v$, and using $p$ as a convenient basepoint, the Abel-Jacobi map (2.4) is given as follows:

$$
\mu(u, v)=\int_{0}^{s} \omega+\int_{0}^{t} \omega
$$

Thus

$$
\frac{\partial \mu}{\partial u}=\frac{s \omega(s)-t \omega(t)}{s-t} \text { and } \quad \frac{\partial \mu}{\partial v}=\frac{-\omega(s)+\omega(t)}{s-t} .
$$

It follows that the Gauss map is given by

$$
\gamma(u, v)=\frac{\omega(s) \wedge \omega(t)}{s-t} .
$$

Since all these expressions are symmetric in $s, t$, they may be written in terms of $u$ and $v$. Putting $p=\omega(0), p^{\prime}=\omega^{\prime}(0), p^{\prime \prime}=\omega^{\prime \prime}(0)$, etc., we compute that

$$
\begin{aligned}
& \gamma(u, v)=p \wedge p^{\prime}+\left[\frac{u}{2} p \wedge p^{\prime \prime}+\frac{v}{2} p^{\prime} \wedge p^{\prime \prime}-\frac{v}{6} p \wedge p^{\prime \prime}\right] \\
&+\left[\frac{u^{2}}{6} p \wedge p^{\prime \prime \prime}+u v\left(\frac{1}{6} p^{\prime} \wedge p^{\prime \prime \prime}-\frac{1}{12} p \wedge p^{(i v)}\right)\right. \\
&\left.\quad+v^{2}\left(\frac{1}{12} p^{\prime \prime} \wedge p^{\prime \prime \prime}+\frac{1}{120} p \wedge p^{(v)}-\frac{1}{24} p^{\prime} \wedge p^{(i v)}\right)\right] \\
&+\left[\frac{u^{3}}{24} p \wedge p^{(i v)}+u^{2} v\left(\frac{1}{24} p^{\prime} \wedge p^{(i v)}-\frac{1}{40} p \wedge p^{(v)}\right)\right. \\
&+u v^{2}\left(\frac{1}{48} p^{\prime \prime}\right.\left.\wedge p^{(i v)}-\frac{1}{60} p^{\prime} \wedge p^{(v)}+\frac{1}{240} p \wedge p^{(v i)}\right) \\
&\left.+v^{3}\left(\frac{1}{144} p^{\prime \prime \prime} \wedge p^{(i v)}-\frac{1}{240} p^{\prime \prime} \wedge p^{(v)}\right)\right]+\cdots .
\end{aligned}
$$

If follows that $\partial \gamma / \partial u=\frac{1}{2} p \wedge p^{\prime \prime}+\cdots, \partial \gamma / \partial v=\frac{1}{2} p^{\prime} \wedge p^{\prime \prime}-\frac{1}{6} p \wedge p^{\prime \prime}+\cdots$, and so we see that $2 p \in \Pi \cap \Delta$ if $p$ is a flex point of $C$. To check the converse, we extend our treatment $(2.10)$ of the second fundamental form to the diagonal.

(2.14) Proposition. At a point $2 p \in \Delta$, with the local coordinates $u, v$ as above, the second fundamental form II is given by the matrix

$$
\mathbf{I I}=\left[\begin{array}{cc}
0 & \frac{1}{2} p \wedge p^{\prime} \wedge p^{\prime \prime} \\
\frac{1}{2} p \wedge p^{\prime} \wedge p^{\prime \prime} & -\frac{1}{6} p \wedge p^{\prime} \wedge p^{\prime \prime \prime}
\end{array}\right] .
$$

Proof. We note from (2.11) that $T_{2 p} \Theta \cong \operatorname{Span}\left(p, p^{\prime}\right) \subset T_{0} J(C)$. In particular, $\partial / \partial u$ and $\partial / \partial v$ correspond to $p$ and $-p^{\prime}$ respectively. The result follows from (2.13) and an argument analogous to the proof of (2.10).

To obtain a local equation for $\Pi$, we need power series expansions for the entries of II as functions of $u, v$. Using (2.11) and (2.13), one computes the leading terms in the equation of $\Pi$ to be as follows:

$$
A+B u+v\left(C+D v+E u^{2}+\cdots\right)+\cdots=0
$$

where

(i) if $p$ is not a flex point, $A, B \neq 0$;

(ii) if $p$ is an ordinary flex point, $A=B=0, C \neq 0$;

(iii) if $p$ is a higher flex point, $A=B=C=0, D=E \neq 0$. 
In particular, we now understand better the nature of the singularities of $\Pi$. The point $2 p \in \Pi$ if and only if $p$ is a flex point of $C$. If $C$ has no higher flex points, then $\Pi$ has no singular points on $\Delta$; thus $\Pi^{\prime}=\Sigma^{2}(\gamma)$ consists only of the bitangent pairs $p+q$ with $p \neq q$. If $C$ has higher flex points $p$, then the points $2 p$ are tacnodes of $\Pi$ (i.e., $\Pi$ has local analytic germ isomorphic to $y^{2}-x^{2} y=0$ ).

Finally, if $p$ is an ordinary flex point of $C$, then the tangent line to $C$ at $p$ cuts $C$ in $3 p+q, p \neq q$, and $l(2 p)=p+q$. Since $\gamma$ is invariant under the involution $l$ and ker $d \gamma$ has nondegenerate tangency with $\Pi$ at $p+q$, it follows that ker $\gamma$ has nondegenerate tangency with $\Pi$ at $2 p$. Thus $(2.8)$ holds at $2 p \in C^{(2)}$, and the proof of $(2.8)$ is complete.

Let $C$ be a smooth plane quartic with no higher flexes. We next compute the number of points of $\Pi^{\prime}$ and $\mathfrak{C}$, reproving the classical theorem that $C$ has 28 bitangents and 24 flexes. The approach is analogous to that in [15], except that we are forced by the presence of singularities in $\Pi$ to work on the normalization $\widetilde{\Pi}$, which we identify with the proper transform of $\Pi$ in $\widetilde{\Theta}$, the blow-up of $\Theta$ at $\Pi^{\prime}$. $\widetilde{\Pi}$ is canonically isomorphic to the Nash blow-up $\widehat{\Pi} \subset \mathbf{P} T \Theta \mid \Pi, \widehat{\Pi}=$ closure $\left\{\left(x, T_{x} \Pi\right): x \in \Pi-\Pi^{\prime}\right\}$. The Nash tangent bundle $\widehat{T} \Pi$ is the restriction to $\widehat{\Pi}$ of the tautological line bundle on $\mathbf{P} T \Theta$. $\widetilde{\Pi}$ is also canonically isomorphic to the kernel blow-up $\Pi^{\#} \subset \mathbf{P} T \Theta \mid \Pi, \Pi^{\#}=\operatorname{closure}\left\{\left(x, \operatorname{ker} d \gamma_{x}\right): x \in \Pi-\Pi^{\prime}\right\}$. The kernel bundle $\mathscr{L}$ is the restriction to $\Pi^{\#}$ of the tautological line bundle on $\mathbf{P} T \Theta$.

The natural map $\pi: \Pi-\Pi^{\prime} \rightarrow C, \pi(p+q)=p$, where $\overline{p q}$ is tangent to $C$ at $p$, extends holomorphically to $\widetilde{\Pi}$; the new map, which we also call $\pi$, is a double cover ramified only at the pairs of points of $\widetilde{\Pi}$ lying over the nodes of $\Pi$. This follows immediately from the local formula given earlier for $\gamma$ near a double point of $\Pi$. Denote then by $\mathscr{R}$ the ramification cycle of the map $\pi$. Let $\delta$ denote the number of nodes of $\Pi$ (equal to the number of bitangents of $C)$, so that $\operatorname{deg} \mathscr{R}=2 \delta$. We now prove the

(2.15) Proposition. $\delta=28$.

Proof. First note that $K_{\Theta} \cong \Theta$. This follows from the adjunction formula [9, p. 147], since the abelian variety $J(C)$ has trivial tangent bundle. Also $\Pi \in|4 \Theta|$. For $\Pi=\{\operatorname{det} \mathbf{I I}=0\}$, and II is a section of $\operatorname{Sym}^{2} T^{*} \boldsymbol{\Theta} \otimes N_{\boldsymbol{\Theta}}$; therefore $\operatorname{det} \mathbf{I I}$ is a section of $K_{\Theta}^{2} \otimes N_{\Theta}^{2} \cong 4 \Theta$. From Riemann-Hurwitz we infer that

$$
\chi\left(\Pi^{\#}\right)=2 \chi(C)-2 \delta=-2 \delta-8 .
$$

On the other hand, we can use the adjunction formula for $\widetilde{\Pi} \subset \widetilde{\Theta}$ to compute $\chi\left(\Pi^{\#}\right)$. Let $E$ denote the exceptional divisor of this blow-up. It is a standard fact about blowing up smooth surfaces $\left[9\right.$, pp. 187, 474-475] that $K_{\widetilde{\Theta}}=\Theta+E$, and $[\widetilde{\Pi}]=[\Pi]-2 E$. From the adjunction formula,

$$
K_{\widetilde{\Pi}}=(\Theta+E)+(4 \Theta-2 E)=5 \Theta-E
$$

on $\tilde{\Pi}$. Thus

$$
-\chi(\widetilde{\Pi})=K_{\widetilde{\Pi}} \cdot \widetilde{\Pi}=(5 \Theta-E) \cdot(4 \Theta-2 E)=20 \Theta^{2}+2 E^{2}=120-2 \delta .
$$


$\left(\Theta^{2}=6\right.$, since $\gamma$ pulls back the hyperplane class on $\mathbf{P}^{2 *}$ to the normal bundle of $\Theta$ and $\gamma$ has degree six.) Now combining this with (2.16) yields the result that $\delta=28$.

We now proceed to compute the divisor class of $\mathfrak{C}$. Let $\eta$ be the degree of $\mathfrak{C}$ (twice the number of flexes of $C$ ).

(2.18) Proposition. $[\mathfrak{C}]=\frac{11}{2} \Theta-\frac{3}{2} \mathscr{R}$ on $\Pi^{\#}$; thus $\eta=48$.

Proof. Interpreting the second fundamental form $\Pi$ as a section of

$$
\operatorname{Hom}\left(T \Theta, T^{*} \Theta \otimes N_{\Theta}\right) \text {, }
$$

we obtain the following exact sequence of vector bundles on $\Pi-\Pi^{\prime}$ :

$$
0 \rightarrow \mathscr{L} \rightarrow T \Theta \stackrel{\text { II }}{\longrightarrow} \operatorname{Ann}(\mathscr{L}) \otimes N_{\Theta} \rightarrow 0 .
$$

The induced bundle map on $\widetilde{\Pi}$ mapping $T \Theta / \mathscr{L} \rightarrow \operatorname{Ann}(\mathscr{L}) \otimes N_{\Theta}$ ramifies along $\mathscr{R}$; that it ramifies simply can be checked directly from the matrix form of II in (2.10). Thus, by Thom-Porteous,

$$
\begin{aligned}
\mathscr{R} & =c_{1}\left(\operatorname{Ann}(\mathscr{L}) \otimes N_{\Theta}-T \Theta / \mathscr{L}\right)=-2 c_{1}(T \Theta / \mathscr{L})+c_{1}\left(N_{\Theta}\right) \\
& =2 \Theta+2 c_{1}(\mathscr{L})+\Theta=3 \Theta+2 c_{1}(\mathscr{L}) .
\end{aligned}
$$

So $c_{1}(\mathscr{L})=\frac{1}{2} \mathscr{R}-\frac{3}{2} \Theta$. Now we observe that the cusp locus $\mathfrak{C}$ is obtained as the zero divisor of the bundle projection $\mathscr{L} \rightarrow T \Theta / \widehat{T} \Pi$, and these zeros are nondegenerate by $(2.8 \mathrm{~d})$. Thus we must compute $c_{1}\left(\mathscr{L}^{*} \otimes T \Theta / \widehat{T} \Pi\right)=$ $-c_{1}(\mathscr{L})+c_{1}(T \Theta)-c_{1}(\widehat{T} \Pi)$. Since $\widehat{T} \Pi \cong T \widetilde{\Pi}$ and $\mathscr{R}=E \cdot \widetilde{\Pi}$, we have by (2.17) that $c_{1}(\widehat{T} \Pi)=-5 \Theta+\mathscr{R}$. Thus the divisor class of $\mathfrak{C}$ on $\widetilde{\Pi}$ is $\frac{11}{2} \Theta-\frac{3}{2} \mathscr{R}$. To compute its degree, we dot with $4 \Theta$, and use the fact that $\operatorname{deg} \mathscr{R}=2 \delta=56$, obtaining $\operatorname{deg} \mathfrak{C}=11 \times 12-3 \times 28=48$.

Hyperelliptic curves. Much the same approach, based on the formula (2.5) for the Gauss map, applies in the hyperelliptic case to prove $(2.1 \mathrm{~b})$. When $C$ is hyperelliptic, the canonical map $\phi: C \rightarrow \mathbf{P}^{2}(2.3)$ gives a two-to-one mapping of $C$ to a conic; the general line $l$ meets $\phi(C)$ in two points, and so the Gauss map has degree four. More interestingly, the theta divisor acquires a singularity, as the Abel-Jacobi map $\mu: C^{(2)} \rightarrow J(C)$ (2.4) collapses the hyperelliptic linear system $E$ to a point $(p+q \in E$ if and only if $p+q$ is a fiber of $\phi$; note that $E$ is a $g_{2}^{1}$, i.e., the linear system has dimension 1 and degree 2). It follows from Riemann's theorem that $\mu\left(C^{(2)}\right)=\Theta$, and from the RiemannKempf singularity theorem that $\mu(E)=x_{0}$, the unique singular point of $\Theta$, is an ordinary node. Indeed, the Abel-Jacobi mapping $\mu: C^{(2)} \rightarrow \Theta$ is the Nash blowup of $\Theta$. By Riemann-Hurwitz, $\phi$ has eight (simple) branch points $P_{1}, P_{2}, \ldots, P_{8}$; these are the eight Weierstrass points of $C$, each of weight three (cf. [9, pp. 273-274]).

Now the formula (2.5) fails to make sense along $E$, but the (Nash) Gauss map $\hat{\gamma}: \widehat{\Theta} \cong C^{(2)} \rightarrow \mathbf{P}^{2 *}$ is given as follows:

$$
\hat{\gamma}(p+q)= \begin{cases}\phi(p) \wedge \phi(q), & \text { if } p+q \notin E, \\ \phi(p) \wedge \phi^{\prime}(q), & \text { if } p+q \in E, \text { and } p \text { is not a Weierstrass point } \\ \phi(p) \wedge \phi^{\prime \prime}(p), & \text { if } p \text { is a Weierstrass point. }\end{cases}
$$


We now proceed to analyze the Thom-Boardman loci of the Gauss map $\hat{\gamma}$. The natural involution $\hat{\imath}$ on $\hat{\Theta}$, induced by the $(-1)$-map of $J(C)$, is given as follows: if $p, q \in C$, let $\bar{p}, \bar{q}$ be the unique points so that $p+\bar{p}$ and $q+\bar{q}$ are the fibers of $\phi$ (if $p$ is a Weierstrass point, then of course $p=\bar{p}$ ); now define $\hat{\imath}: \widehat{\Theta} \rightarrow \widehat{\Theta}$ by $\hat{\imath}(p+q)=\bar{p}+\bar{q}$. Then $x \in \widehat{\Theta}$ is a fixed point of $\hat{\imath}$ if and only if $x \in E$ or $x=p+q, p \neq q$, where both $p$ and $q$ are Weierstrass points.

We prove Theorem $(2.1 \mathrm{~b})$ by using $(2.19)$ to describe the geometry of the parabolic curve $\Pi=\bar{\Sigma}^{1}(\hat{\gamma})$ and the loci $\Pi^{\prime}=\Sigma^{2}(\hat{\gamma})$ and $\mathfrak{C}=\Sigma^{1,1}(\hat{\gamma})$ in the Nash blow-up $\widehat{\Theta}$ of the theta divisor. Let $\Gamma_{j}$ denote the curve $\left\{P_{j}\right\} \times C \subset C^{(2)}$, $j=1,2, \ldots, 8$. Observe that $\Gamma_{j} \cap \Delta=\Gamma_{j} \cap E=\left\{2 P_{j}\right\}$.

(2.20) Theorem. Let $C$ be a smooth hyperelliptic curve, and let $\hat{\gamma}$ be the Nash Gauss map on $\widehat{\Theta}$.

(a) $\Pi=E+\Sigma \Gamma_{j}$;

(b) $\mathfrak{C}=\Sigma^{1,1,1}(\hat{\gamma})=\left\{2 P_{j}\right\} \subset \Delta$.

(c) $\Pi^{\prime}=\left\{P_{i}+P_{j}: i \neq j\right\}$;

(d) $\Pi$ is smooth except for ordinary nodes at $\Pi^{\prime}$ and $\mathfrak{C}$. At each point $x$ of $\mathfrak{C}$, one branch of $\Pi$ is pointwise fixed by the involution, and $\operatorname{ker} d \hat{\gamma}_{x}$ has nondegenerate tangency at $x$ with the other branch.

Remarks. Theorem (2.1b) follows immediately from (2.20) and the transversality package (1.7). We find one surprise, namely the presence of "exotic" cusps. In particular, $\Sigma^{1,1,0}=\varnothing$, and the cusp locus emerges as the Thom-Boardman locus $\Sigma^{1,1,1}$.

Proof. Outside the curve $E \subset \widehat{\Theta}$, the local analysis of the singularities of $\hat{\gamma}$ is the same as in the nonhyperelliptic case, including that at $\Pi^{\prime}$. However, since $\hat{\imath}$ fixes $E$ pointwise, the singularities of $\hat{\gamma}$ have type $(R \rightarrow T)$ at points of $E$.

First we observe that on $C^{(2)}-\Delta-E$, using local coordinates $s, t$ near $p, q$ respectively,

$$
d \hat{\gamma}(p+q)=\phi^{\prime}(p) \wedge \phi(q) d s+\phi(p) \wedge \phi^{\prime}(q) d t \quad \bmod (\phi(p) \wedge \phi(q)) .
$$

Since a chord of a smooth plane conic can be nowhere tangent to the conic, $d \hat{\gamma}$ will be singular only if $\phi^{\prime}(p)$ or $\phi^{\prime}(q)$ is zero. Thus we fine that $\hat{\gamma}$ has rank one along the curves $\Gamma_{j}$ and rank zero at the points $P_{i}+P_{j}, i \neq j$. It is moreover immediate from (2.21) that the only critical points of $\hat{\gamma} \mid\left(\Gamma_{j}-\left\{2 P_{j}\right\}\right)$ are the points $P_{i}+P_{j}, i \neq j$; these cannot belong to $\Sigma^{1,1}$, of course, since $\Sigma^{1,1} \cap \Sigma^{2}=$ $\varnothing$. So we conclude that $\Gamma_{j} \subset \Pi, j=1,2, \ldots, 8$, and $\left\{P_{i}+P_{j}: i \neq j\right\} \subset \Pi^{\prime}$. Moreover, there are no further points of $\Pi$ or $\Pi^{\prime}$, and no points of $\mathfrak{C}$, in the complement of $\Delta \cup E$.

Consider next the behavior of $\hat{\gamma}$ along $\Delta$. Away from $\Delta \cap E=\left\{2 P_{j}\right\}$, the formula (2.13) for the Gauss map holds, provided we interpret $p^{(i)}$ as $\phi^{(i)}(p)$. Since a conic has no flex points, it follows that $\hat{\gamma}$ has maximal rank along $\Delta-E$.

The most interesting analysis occurs at points of $E$. Given $p+q \in E-\Delta$, we may choose local coordinates $s, t$ near $p, q$ respectively so that $E=\{s=t\}$; e.g., use the local covering map $\phi$ to pull back a local coordinate on the conic. Since by definition $\phi(p)=\phi(q)$ for $p+q \in E$, the resulting power series expansion (2.12) for $\hat{\gamma}$ is divisible by $s-t$ (this reflects the fact that $E$ is the base locus of the map $\gamma$ ). Upon dividing, we obtain an expression symmetric in $s, t$; it follows that the map has rank at most one along $\{s=t\}$. Thus, $E \subset \Pi$, 
completing the proof of $(2.21 \mathrm{a})$. That $E-\Delta \subset \Sigma^{1,0}(\hat{\gamma})$ can be checked from the expression for $\hat{\gamma}$ to which we refer, or by parametrizing the conic explicitly: $\phi(s)=\left(1, s, s^{2}\right), \phi(t)=\left(1, t, t^{2}\right)$, whence $\hat{\gamma}(s, t)=(s t, s+t)$. (This differs by a linear change of coordinates from the normal form for a symmetric fold given in (1.6)). Thus $\Pi^{\prime} \cap(E-\Delta)=\varnothing$ and $\mathfrak{C} \cap(E-\Delta)=\varnothing$.

Now consider $P=P_{j}, 2 P \in \Delta \cap E$. Here we choose local coordinates $s, t$ and $P$ on $C$ so that $E=\{s+t=0\}$; e.g., choose $s, t$ and a local coordinate $z$ centered at $\phi(P)$ on the conic so that $\phi$ is given by $s^{2}=z=t^{2}$. Let $u, v$ be the canonical symmetric coordinates in $s, t$ as usual, $u=s+t, v=s t$. Then in these coordinates, $E=\{u=0\}$ and $\Gamma_{j}=\{v=0\}$. Examining the formula (2.13) for the Gauss map in the symmetric variables, we must be somewhat prudent since $\phi^{\prime}(P)=0$. The crucial observation is this: every term in this expansion is divisible by $u$. (To see why, think of $u, v$ as being variables of weights 1,2 respectively; the coefficient of $p^{(j)} \wedge p^{(k)}$ has weight $j+k-1$, as is easily verified. Now it follows that any pure- $v$ term, being of even weight, appears as a coefficient of $p^{(j)} \wedge p^{(k)}$ where $j+k$ is odd. Therefore either $j$ or $k$ must be odd, and any odd derivative of $\phi$ vanishes at $P$.) Finally we may conclude, upon dividing the resulting formula $(2.12)$ by $u$, that

$$
\begin{aligned}
\hat{\gamma}(u, v)= & p \wedge p^{\prime \prime}+\left(\frac{u^{2}}{24}-\frac{v}{12}\right) p \wedge p^{(i v)} \\
& +v^{2}\left(\frac{1}{48} p^{\prime \prime} \wedge p^{(i v)}+\frac{1}{240} p \wedge p^{(v i)}\right)+\cdots .
\end{aligned}
$$

Note that $\hat{\gamma} \mid \Gamma_{j}$ drops rank at $2 P$, while $\hat{\gamma} \mid E$ does not. (It is immediate from (2.22) that the kernel vector $\partial / \partial u$ is simply tangent to $\Gamma_{j}$ at $2 P_{j}$. In addition, it is worth observing that $\hat{\gamma} \mid E$ maps $E$ isomorphically to the dual conic of $\phi(C)$.) Near the point $2 P$, the map $\hat{\gamma}$ has the local form $(u, v) \rightarrow\left(u^{2}-2 v\right.$, $\left.v^{2}\right)$. This is most easily seen by taking the explicit parametrization $\phi(t)=$ $\left(1, t^{2}, t^{4}\right)$ of the canonical map at the Weierstrass point $P$. (It also follows from (2.22), once one checks that the two vectors $p \wedge p^{(i v)}$ and $5 p^{\prime \prime}+p \wedge p^{(v i)}$ are linearly independent.) Once again, this differs only by a simple change of variables from the normal form for a symmetric cusp given in (1.6). Thus $E \cap \Delta \subset \mathfrak{C}$, and (2.20d) holds. This concludes the proof of Theorem (2.20) and of Theorem (2.1b).

The Gauss map as a deformation of its fibers. An alternate approach to the stability of the Gauss map is to consider the singularities of its fibers. Let $C$ be a smooth nonhyperelliptic curve of genus 3 , and let $\gamma: \Theta \rightarrow \mathbf{P}^{2 *}$ be the Gauss map of the theta divisor of $J(C)$. If $x \in \Theta$ and $l=\gamma(x)$, the fiber $(F, x)=\left(\gamma^{-1}(l), x\right)$ is a local analytic $(Z / 2)_{x}$-space (recall from $\S 1$ that $(\mathbf{Z} / 2)_{x}$ denotes the subgroup of $\mathbf{Z} / 2$ fixing $\left.x\right)$. Being a finite map between smooth spaces of the same dimension, $\gamma$ is a flat map [10, Example 9.3, p. 266]. Thus, by (1.5), the germ of $\gamma$ at $x$ is $(\mathbf{Z} / 2)_{x}-V$-stable if and only if it is a $(\mathbf{Z} / 2)_{x}$-versal deformation of $(F, x)$, i.e., if and only if the Kodaira-Spencer map

$$
\varphi: T_{\gamma(x)} \mathbf{P}^{2 *} \rightarrow\left(T_{(F, x)}^{1}\right)^{(\mathbf{Z} / 2)_{x}}
$$

is surjective. Theorem (2.7) therefore follows from the statement that, for a smooth plane quartic $C$, the germ of $\gamma$ at $x$ is a $(Z / 2)_{x}$-versal deformation of $(F, x)$ if and only if $x \neq 2 p$ for $p$ a higher flex of $C$. This statement 
can be established through a case-by-case analysis of the different types of fibers $(F, x)$.

To carry out this analysis, we again identify the theta divisor with the symmetric square $C^{(2)}$ of $C$ (cf. (2.4)); $\gamma(p+q)$ is the secant line of $C$ through $p$ and $q$, and $\gamma(2 p)$ is the tangent line of $C$ at $p$. Since $C$ has degree four, each fiber of $\gamma$ is a scheme of length six. We list below the five different types of fibers $F=\gamma^{-1}(l)$. If $x, y \in \Theta$ and $l(x)=y$, we write $x \leftrightarrow y$. We assume $p, q, r, s$ are distinct points of $C$.

(2.23) Fibers of the Gauss map ( $C \subset \mathbf{P}^{2}$ quartic curve).

\begin{tabular}{lllll} 
& \multicolumn{1}{c}{$l \cap C$} & points of $F$ & multiplicity & \\
(i) & $p+q+r+s$ & $p+q \leftrightarrow r+s$ & 1 & \\
& & $p+r \leftrightarrow q+s$ & 1 & \\
& & $p+s \leftrightarrow q+r$ & 1 & \\
(ii) & $2 p+q+r$ & $2 p \leftrightarrow q+r$ & 1 & \\
& & $p+q \leftrightarrow p+r$ & 2 & (a) \\
(iii) $3 p+q$ & $p+q \leftrightarrow 2 p$ & 3 & (b) \\
(iv) $2 p+2 q$ & $2 p \leftrightarrow 2 q$ & 1 & \\
& & $p+q$ & 4 & (c) \\
(v) $4 p$ & $2 p$ & 6 & (d)
\end{tabular}

From this table we see that $(F, x)$ is singular (i.e., $x$ has multiplicity at least 2 in $F$ ) if and only if $x=p+q$, with $\overline{p q}$ tangent to $C$ at $p$, or $x=2 p$, with $p$ a flex of $C$. These are the cases labeled (a), (b), (c), (d) above. If $x$ has type (a) or (b), then $(\mathbf{Z} / 2)_{x}$ is trivial; if $x$ has type (c) or $(d)$, then $(\mathbf{Z} / 2)_{x}=\mathbf{Z} / 2$, and $x$ is an isolated fixed point of $l$.

From this point of view, Theorem (2.8) takes the following form.

(2.24) Theorem. Let $C$ be a smooth plane quartic. For $x \in C^{(2)}$ of type (2.23) (a), (b), (c), (d), the fiber $(F, x)$ has type $A_{1}, A_{2}, F^{2,2}, F^{2,4}$, respectively. For types $(\mathbf{a}),(\mathbf{b}),(\mathrm{c}),(F, x)$ is $(\mathbf{Z} / 2)_{x}$-versally deformed by $\gamma ;$ for type $(\mathrm{d}),(F, x)$ is not $(\mathbf{Z} / 2)_{x}$-versally deformed by $\gamma$.

A proof of (2.24) independent of (2.8) can be given using local parametrizations $p(s), q(t)$ of $C$ to compute local equations for $\gamma$. The geometry of the fibers of $\gamma$ can be used to simplify computations. We give here the details when $x$ has type $(2.23)(\mathrm{c})$. Then $x=p+q$, with $p \neq q$, and $\overline{p q}$ is bitangent to $C$ at $p$ and $q$.

We take $p=p(s)=\left(1, s, s^{2}+\cdots\right)$ and $q=q(t)=\left(t, 1, t^{2}+\cdots\right)$, so $\gamma(s, t)=\left(s^{2}+\cdots, t^{2}+\cdots\right)$ in affine coordinates. We claim that the ideal $I=\left\langle s^{2}+\cdots, t^{2}+\cdots\right\rangle$ defining $x$ in $\mathbf{C}[[s, t]]$ is equal to the ideal $\left\langle s^{2}, t^{2}\right\rangle$. Let $A=C[[s, t]] / I$. Since the fiber $F$ of $\gamma$ has total length 6 and consists of $(F, x)$ and two reduced points, $\operatorname{dim}_{\mathbf{C}} A=4$. Let $\mathbf{m}=\langle s, t\rangle$ be the maximal ideal at $0 ;$ since $\operatorname{dim}\left(A / \mathbf{m}^{3} A\right)=4$, we must have $\mathbf{m}^{3} A=0$. Therefore, $I=$ $I+\mathbf{m}^{3}=\left\langle s^{2}+\cdots, t^{2}+\cdots\right\rangle+\langle s, t\rangle^{3}=\left\langle s^{2}, t^{2}\right\rangle$. Thus $(F, x)$ is an $F^{2,2}$ singularity (cf. $[14$, p. 130]).

Let $T^{1}=T_{(F, x)}^{1}$ and $\left(T^{1}\right)^{+}=\left(T_{(F, x)}^{1}\right)^{\mathrm{Z} / 2}$. It is easy to compute that $\operatorname{dim}_{\mathbf{C}} T^{1}=4$, with graded quotients $T^{1} / \mathbf{m} T^{1}$ and $\mathbf{m} T^{1} / \mathbf{m}^{2} T^{1}$ both of dimension $2 ; T^{1} / \mathbf{m} T^{1}$ has basis $(1,0),(0,1)$. Since $\mathbf{Z} / 2$ acts on the germ $\left(C^{(2)}, x\right) \cong\left(\mathbf{C}^{2}, 0\right)$ with the origin as an isolated fixed point, the induced action on the cotangent space $\mathbf{m} / \mathbf{m}^{2}$ is $-\mathrm{id}$. Thus the induced action on the 
graded quotient $\mathbf{m}^{k} T^{1} / \mathbf{m}^{k+1} T^{1}$ of $T^{1}$ is $(-1)^{k}$ id, so $\left(T^{1}\right)^{+}$has dimension 2, with basis $(1,0),(0,1)$; i.e., the Kodaira-Spencer map is surjective. In other words, the germ of $\gamma$ at $x$ is a $\mathbf{Z} / 2$-versal deformation of $(F, x)$.

(2.25) Remark. The proof of (2.24) shows that if $F=\gamma^{-1}(l)$ contains no points of type (d), then the analytic $\mathbf{Z} / 2$-space $F$ is $\mathbf{Z} / 2$-versally deformed by $\gamma$. (The reason is that $F$ contains at most one $\mathbf{Z} / 2$-orbit of singular points.) Thus, if $C$ is a smooth plane quartic with no higher flexes, then the Gauss map of the theta divisor is a $\mathbf{Z} / 2$-versal deformation of each of its fibers.

A similar analysis can be used for hyperelliptic curves. Let $C$ be a smooth hyperelliptic curve of genus 3, and let $\hat{\gamma}: \widehat{\Theta} \rightarrow \mathbf{P}^{2 *}$ be the (Nash) Gauss map of the theta divisor of $J(C)$. If $x \in \widehat{\Theta}$ and $l=\hat{\gamma}(x)$, the fiber $(F, x)=$ $\left((\hat{\gamma})^{-1}(l), x\right)$ is a local analytic $(\mathbf{Z} / 2)_{x}$-space, and it can be checked directly that the germ of $\hat{\gamma}$ at $x$ is a $(\mathbf{Z} / 2)_{x}$-versal deformation of $(F, x)$ for all $x \in \widehat{\Theta}$ (and in fact $\hat{\gamma}$ is a $\mathbf{Z} / 2$-versal deformation of each fiber $F$ ). This gives a proof of $(2.1 \mathrm{~b})$ complementary to the approach taken in (2.20).

For this proof, we identify $\widehat{\Theta}$ with $C^{(2)}$; if $p+q$ is not in $E$ and $p \neq q$, then $\hat{\gamma}(p+q)$ is the line through $\phi(p)$ and $\phi(q)$ (cf. (2.20)). Each fiber $F=(\hat{\gamma})^{-1}(l)$ has length 4 . The possible fibers are given below. In this table, $\phi^{-1}(a)=p+\bar{p}$, $\phi^{-1}(b)=q+\bar{q}, \phi^{-1}(A)=2 p$, and $\phi^{-1}(B)=2 q$.

(2.26) Fibers of the Gauss map $\left(\phi: C \rightarrow \mathbf{P}^{2}\right.$ double cover of a conic).

$\begin{array}{lllc} & \ln \phi(C) & \text { points of } F & \text { multiplicity } \\ \text { (i) } & a+b & p+q \leftrightarrow \bar{p}+\bar{q} & 1 \\ & & p+\bar{q} \leftrightarrow \bar{p}+q & 1 \\ \text { (ii) } & A+b & p+q \leftrightarrow p+\bar{q} & 2 \\ \text { (iii) } & A+B & p+q & 4 \\ \text { (iv) } & 2 a & 2 p \leftrightarrow 2 \bar{p} & 1 \\ & & p+\bar{p} & 2 \\ \text { (v) } & 2 A & 2 p & 4\end{array}$

\section{SPECIALIZATION TO THE HYPERELLIPTIC CASE}

Let $\mathscr{A}_{3}$ be the moduli space of 3-dimensional principally polarized abelian varieties $(A, \Theta)$ (cf. [22]). The Gauss map of the theta divisor is the rational map $\gamma: \Theta \rightarrow \mathbf{P} T_{0}^{*}(A)=\mathbf{P}^{2 *}$ which assigns to a smooth point $x$ the translate of the tangent plane $T_{x} \Theta$ to the origin of the abelian variety $A$. The map $x \rightarrow-x$ on $A$ induces an involution $l$ of $\Theta$, and $\gamma$ is invariant under this $\mathbf{Z} / 2$-action. We say that the theta divisor $\Theta$ is Gauss-stable if $\Theta$ is smooth and the Gauss map $\gamma: \Theta \rightarrow \mathbf{P}^{2 *}$ is locally $\mathbf{Z} / 2$-stable; note that this is a slight change in terminology from the previous section.

(3.1) Theorem. The set of 3-dimensional principally polarized abelian varieties with Gauss-stable theta divisor is a nonempty Zariski-open subset of $\mathscr{A}_{3}$.

Proof. The Torelli map $\mathscr{M}_{3} \rightarrow \mathscr{A}_{3}$, which assigns to a curve $C$ its Jacobian variety, is an isomorphism of $\mathscr{M}_{3}$ with the Zariski-open subset of $\mathscr{A}_{3}$ consisting of abelian varieties with irreducible theta divisor. The image of the nonhyperelliptic curves under this map is $\mathscr{A}_{3}-\mathscr{N}_{0}$, where $\mathscr{N}_{0}$ is the locus of abelian varieties with singular theta divisor. Since $\mathscr{N}_{0}$ is a proper Zariski-closed subset of $\mathscr{A}_{3}$, the theorem follows from the stability theorem for curves (2.2). 
In this section we give another proof of Theorem (3.1). The above proof relies on the realization of the generic 3-dimensional principally polarized abelian variety as the Jacobian of a genus 3 curve. Here we present a "dynamic" proof using deformation theory. The advantage of this method of proof is that it may apply in higher dimensions, where the generic abelian variety is not a Jacobian.

Let $\mathscr{G}$ be the set of 3 -dimensional principally polarized abelian varieties $(A, \boldsymbol{\Theta})$ such that $\boldsymbol{\Theta}$ is Gauss-stable. First we prove in (3.12) that $\mathscr{G}$ is Zariskiopen in $\mathscr{A}_{3}$ for rather general reasons: Since the Gauss map is a $Z / 2$-versal deformation of a fiber if and only if the Kodaira-Spencer map is surjective, $\mathscr{G}$ is the complement of the support of a certain cokernel sheaf.

To show that $\mathscr{G}$ is nonempty we use a variational analysis: specialization to a hyperelliptic theta divisor. We start with a generic one-parameter family of theta divisors $\Theta_{t}$ specializing to a hyperelliptic theta divisor $\Theta_{0}$. We blow up the total space of the family at the singular point of $\Theta_{0}$ so that the relative Gauss map $\gamma_{t}$ is defined for all $t$. After base change and normalization, we obtain in (3.13) a family $\mathscr{X}$ such that $\Theta_{0}$ is replaced by a new central fiber $X_{0}$ for which we can explicitly calculate the fibers of the Gauss map and their induced first-order deformations. We compute the contribution that the "horizontal" variation $\left.(d / d t)\right|_{t=0}$ makes to the cokernel of the Kodaira-Spencer map. We deduce that the Kodaira-Spencer map is surjective for $t \neq 0$. Thus the nearby Gauss maps $\gamma_{t}$, for $t \neq 0$, are $\mathbf{Z} / 2$-versal deformations of their fibers, and hence the maps $\gamma_{t}$ are $\mathrm{Z} / 2$-stable.

The Kodaira-Spencer map. We summarize the results from deformation theory which we use in this proof. Basic references on the Kodaira-Spencer map are $[13,11]$, and the talks by Ruget, Berthelot, and Teissier in [7]. A less general but more geometric treatment is given in [14].

A flat map $f: X \rightarrow Y$ of analytic spaces can be viewed as defining a deformation of each of its fibers. We assume moreover that $Y$ is smooth, and that $X$ has an involution $l$ such that $f \circ l=f$. Let $F_{y}=f^{-1}(y)$. For each $y \in Y$, the image of the Kodaira-Spencer map $T_{y}(Y) \rightarrow T_{F_{y}}^{1}$ lies in the subspace $\left(T_{F_{y}}^{1}\right)^{+}$ of $l$-invariant elements, so we have a Kodaira-Spencer map

$$
\varphi_{y}: T_{y}(Y) \rightarrow\left(T_{F_{y}}^{1}\right)^{+} .
$$

For each $x \in F_{y},(3.2)$ restricts to the local Kodaira-Spencer map $T_{y}(Y) \rightarrow$ $\left(T_{\left(F_{y}, x\right)}^{1}\right)^{+}$of (1.5). If $X$ is smooth and $f$ is finite, then the map (3.2) is surjective if and only if $f$ is a $\mathbf{Z} / 2$-versal deformation of $F_{y}$ (cf. [12, 7, p. 176]).

The maps (3.2) fit together to give a sheaf map

$$
\varphi: \mathscr{T}_{Y} \rightarrow\left(f_{*} \mathscr{T}_{X / Y}^{1}\right)^{+} .
$$

Here $\mathscr{T}_{Y}$ is the tangent sheaf of $Y$, and $\left(\mathscr{T}_{X / Y}^{1}\right)^{+} \mid F_{y}=\left(\mathscr{T}_{F_{y}}^{1}\right)^{+}$, a sheaf with global sections $\left(T_{F_{y}}^{1}\right)^{+}$.

Next we consider a family of invariant maps $f_{t}: X_{t} \rightarrow Y_{t}, t \in S$; that is, a diagram

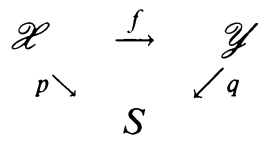


where $f, p, q$ are proper, flat maps of analytic spaces, $p=q \circ f$, and there is an involution $l$ of $X$ such that $p \circ l=p$ and $f \circ l=f$. We make the following additional assumptions:

(a) $\mathscr{X}, \mathscr{Y}, S$ are smooth,

(b) $f$ is finite,

(c) $q$ is smooth (submersive).

In this situation there is a "relative" Kodaira-Spencer map

$$
\varphi: \mathscr{T}_{\mathscr{Y} / S} \rightarrow\left(f_{*} \mathscr{T}_{\mathscr{X} / \mathscr{Y}}^{1}\right)^{+} \text {. }
$$

Here $\mathscr{T}_{\mathscr{Y} / S}$ is the tangent sheaf along the fibers of $q, \mathscr{T}_{\mathscr{X} / \mathscr{Y}}^{1}$ restricts on $X_{t}$ to $\mathscr{T}_{X_{t} / Y_{t}}$, and $\varphi_{t}$ is the map (3.3) for each $t \in S$. Considering $f$ just as a map, we have an "absolute" Kodaira-Spencer map $\Phi$ and a commutative triangle

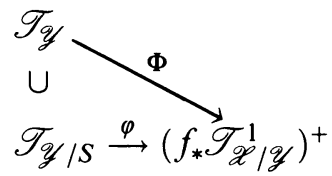

We will need the following special case of the naturality of the KodairaSpencer map. Let $\beta: \mathscr{T}_{S} \rightarrow\left(f_{*} \mathscr{T}_{\mathscr{X} / S}^{1}\right)^{+}$be the Kodaira-Spencer map of the family $p: \mathscr{X} \rightarrow S$. The following square commutes:

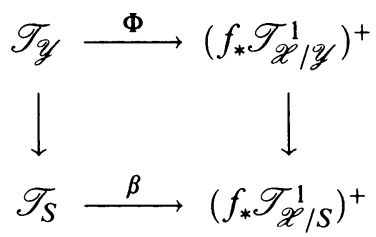

where the vertical arrows are induced by $q: \mathscr{Y} \rightarrow S$.

The above properties of the Kodaira-Spencer map can be proved using the cotangent functors $\mathscr{T}^{i}$ (cf. $[13,11]$ ); by convention, $\mathscr{T}^{0}=\mathscr{T}$. Given a family $f: \mathscr{X} \rightarrow \mathscr{Y}$ over $S$, there is an exact sequence of sheaves on $\mathscr{X}:$

$$
0 \rightarrow \mathscr{T}_{\mathscr{X} \mid \mathscr{Y}}^{0} \rightarrow \mathscr{T}_{\mathscr{X} / S}^{0} \rightarrow f^{*} \mathscr{T}_{\mathscr{Y} / S}^{0} \rightarrow \mathscr{T}_{\mathscr{X} \mid \mathscr{Y}}^{1} \rightarrow \mathscr{T}_{\mathscr{X} / S}^{1} \rightarrow f^{*} \mathscr{T}_{\mathscr{Y} / S}^{1} \rightarrow \cdots,
$$

and the Kodaira-Spencer map $\varphi: \mathscr{T}_{\mathscr{Y} / S} \rightarrow f_{*} \mathscr{T}_{\mathscr{X} / \mathscr{y}}^{1}$ is the composition $\mathscr{T}_{\mathscr{Y} / S}^{0} \rightarrow$ $f_{*} f^{*} \mathscr{T}_{\mathscr{Y} / S}^{0} \rightarrow f_{*} \mathscr{T}_{\mathscr{X} / \mathscr{Y}}^{1}$. If $A \rightarrow B$ is smooth, then $\mathscr{T}_{A / B}^{0}$ is the tangent sheaf along the fibers and $\mathscr{T}_{A / B}^{1}=0$. If $A \rightarrow B$ is a finite map of smooth spaces, then $\mathscr{T}_{A / B}^{0}=0$. Thus, under the assumptions (3.4), we have exact sequences

$$
\begin{gathered}
0 \longrightarrow \mathscr{T}_{\mathscr{X}}^{0} \longrightarrow f^{*} \mathscr{T}_{\mathscr{Y}}^{0} \longrightarrow \mathscr{T}_{\mathscr{X} / \mathscr{Y}}^{1} \longrightarrow 0 \\
\cup \\
\cup \quad \| \\
0 \longrightarrow \mathscr{T}_{\mathscr{X} / S}^{0} \longrightarrow f^{*} \mathscr{T}_{\mathscr{Y} / S} \longrightarrow \mathscr{T}_{\mathscr{X} / \mathscr{Y}}^{1} \longrightarrow \mathscr{T}_{\mathscr{X} / S}^{1} \longrightarrow 0
\end{gathered}
$$

Pushing down to $\mathscr{Y}$ by the finite map $f$, and taking invariant subsheaves under the action of $l$, we get exact sequences

$$
0 \rightarrow\left(f_{*} \mathscr{T}_{\mathscr{X}}^{0}\right)^{+} \rightarrow\left(f_{*} f^{*} \mathscr{T}_{\mathscr{Y}}^{0}\right)^{+} \rightarrow\left(f_{*} \mathscr{T}_{\mathscr{X} \mid \mathscr{Y}}^{1}\right)^{+} \rightarrow \quad 0
$$

$$
\begin{gathered}
\cup \\
0 \rightarrow\left(f_{*} \mathscr{T}_{\mathscr{X} / S}^{0}\right)^{+} \rightarrow\left(f_{*} f^{*} \mathscr{T}_{\mathscr{Y} / S}^{0}\right)^{+} \rightarrow\left(f_{*} \mathscr{T}_{\mathscr{X} / \mathscr{Y}}^{1}\right)^{+} \rightarrow\left(f_{*} \mathscr{T}_{\mathscr{X} / S}^{1}\right)^{+} \rightarrow 0
\end{gathered}
$$


which define $\varphi$ and $\Phi$ (3.6). The naturality of (3.8) [11, 2.1.5, p. 137] implies the naturality (3.7) of the Kodaira-Spencer map.

We apply the above theory to a one-parameter family such that $\mathscr{Z}$ has one singular fiber.

(3.10) Proposition. Let $f: \mathscr{X} \rightarrow \mathscr{Y}$ be a family of maps over the disc $\Delta$ satisfying the assumptions (3.4), and such that $p$ is smooth away from $t=0$. Suppose that for every $y \in Y_{0}$, the Kodaira-Spencer map $\varphi_{y}: T_{y}\left(Y_{0}\right) \rightarrow\left(T_{F_{y}}^{1}\right)^{+}$ is surjective if $X_{0}$ is smooth at all points of $F_{y}$, and $\operatorname{dim}\left(\operatorname{coker}\left(\varphi_{y}\right)\right) \leq 1$ if $X_{0}$ is singular at some point of $F_{y}$. Then there is an $\varepsilon>0$ such that for all $t$ with $0<|t|<\varepsilon$, and for all $y \in Y_{t}, \varphi_{y}$ is surjective.

Since the absolute Kodaira-Spencer map $\Phi: \mathscr{T}_{\mathscr{Y}}^{0} \rightarrow\left(f_{*} \mathscr{T}_{\mathscr{L} / \mathscr{Y}}^{1}\right)^{+}$for the family (3.10) sends $\mathscr{T}_{\mathscr{Y} / \Delta}^{0}$ to $\operatorname{im}(\varphi)$ (by (3.6)), there is an induced map

$$
\bar{\Phi}: q^{*}\left(\mathscr{T}_{\Delta}^{0}\right)=\mathscr{T}_{\mathscr{Y}}^{0} / \mathscr{T}_{\mathscr{Y} / \Delta}^{0} \rightarrow \operatorname{coker}(\varphi) .
$$

(3.11) Lemma. $\left.\bar{\Phi}(d / d t)\right|_{y} \neq 0$ if and only if $y \in Y_{0}$ and $X_{0}$ is singular at some point of $F_{y}$.

In other words, for $y \in Y$, the first order variation over $(\mathscr{Y}, y)$ of the zerodimensional $\mathbf{Z} / 2$-scheme $F_{y}$ is strictly greater than the first order variation over $\left(Y_{t}, y\right)$, where $t=q(y)$, if and only if $X_{0}$ is singular at some point of $F_{y}$.

Proof of (3.10). By (3.11), the hypothesis of (3.10) implies that $\Phi_{y}$ is surjective for all $y \in Y_{0}$. So there exists $\varepsilon>0$ such that for all $t$ with $0<|t|<\varepsilon$, and all $y \in Y_{t}, \Phi_{y}$ is surjective. Again by (3.11), this implies that for all such $t$ and $y, \varphi_{y}$ is surjective.

Proof of (3.11). First we show $\operatorname{Supp}(\bar{\Phi}(d / d t)) \subset\left\{y \in Y_{0}: X_{0}\right.$ is singular at some point of $\left.F_{y}\right\}$. Let $V=\mathscr{Y}-f\left(\operatorname{Sing} X_{0}\right)$ and $U=f^{-1}(V)$. The map $p: U \rightarrow$ $\Delta$ is smooth, so $\mathscr{T}_{\mathscr{X}}^{0}\left|U \rightarrow\left(p^{*} \mathscr{T}_{\Delta}^{0}\right)\right| U$ is surjective. Therefore $\left(f_{*} \mathscr{T}_{\mathscr{X}}^{0}\right)^{+} \mid V \rightarrow$ $\left(f_{*} p^{*} \mathscr{T}_{\Delta}^{0}\right)^{+}\left|V=\left(f_{*} f^{*} q^{*} \mathscr{T}_{\Delta}^{0}\right)^{+}\right| V$ is surjective. So $(d / d t) \mid V$ is in the image of $\left(f_{*} \mathscr{T}_{\mathscr{X}}^{0}\right)^{+} \mid V$ and hence goes to 0 in $\operatorname{coker}(\varphi) \mid V$, by (3.9).

Conversely, suppose $y \in Y_{0}$, and $X_{0}$ is singular at some point of $F_{y}$. To show $\left.\overline{\boldsymbol{\Phi}}(d / d t)\right|_{y} \neq 0$, we show that $\left.\alpha(\bar{\Phi}(d / d t))\right|_{y} \neq 0$, where $\alpha: \operatorname{coker}(\varphi) \rightarrow$ $f_{*} \mathscr{T}_{\mathscr{X} / \Delta}^{1}$ is induced by the homomorphism $\left(f_{*} \mathscr{T}_{\mathscr{X} / \mathscr{Y}}^{1}\right)^{+} \rightarrow f_{*} \mathscr{T}_{\mathscr{X} / \Delta}^{1}$ of (3.9). By naturality (3.7), $\alpha \circ \bar{\Phi}=\beta$, where $\beta: q^{*}\left(\mathscr{T}_{\Delta}^{0}\right) \rightarrow f_{*} \mathscr{T}_{\mathscr{Z} / \Delta}^{1}$ is induced by the Kodaira-Spencer map of the family $\mathscr{X} \rightarrow \Delta$. It remains to show that $\left.\beta(d / d t)\right|_{y} \neq 0$. Since $\mathscr{X}$ is smooth, $\mathscr{T}_{\mathscr{X}}^{1}=0$, so there is an exact sequence

$$
0 \rightarrow f_{*} \mathscr{T}_{\mathscr{L} / \Delta}^{0} \rightarrow f_{*} \mathscr{T}_{\mathscr{X}}^{0} \rightarrow f_{*} p^{*} \mathscr{T}_{\Delta}^{0} \rightarrow f_{*} \mathscr{T}_{\mathscr{X} / \Delta}^{1} \rightarrow 0 .
$$

Now $d / d t$ generates $f_{*} p^{*} \mathscr{T}_{\Delta}^{0}$ as an $f_{*} \mathscr{O}_{\mathscr{L}}$-module, so $\beta(d / d t)$ generates $f_{*} \mathscr{T}_{\mathscr{L} / \Delta}$ as an $f_{*} \mathscr{O}_{\mathscr{L}}$-module. Therefore the support of $\beta(d / d t)$ equals the support of the $\mathscr{O}_{\mathscr{y}}$-module $f_{*} \mathscr{T}_{\mathscr{X} / \Delta}^{1}$. But $\operatorname{Supp}\left(f_{*} \mathscr{T}_{\mathscr{X} / \Delta}^{1}\right)=f\left(\operatorname{Sing} X_{0}\right)$.

The dynamic proof of the stability theorem. For the Gauss map $\gamma: \Theta \rightarrow \mathbf{P}^{2 *}$ of a nonhyperelliptic theta divisor, the sheaf $\mathscr{T}_{F_{\mathrm{v}}}^{1}$ is supported on at most one $\mathrm{Z} / 2$-orbit. (Indeed, $F_{y}$ has total length 6 , length $\geq 2$ at a singular point, and 
length $\geq 4$ at a fixed point of the involution $l$.) Therefore, for all $y \in \mathbf{P}^{2 *}$, $\gamma$ is a $\mathbf{Z} / 2$-versal deformation of $F_{y}$ if and only if, for all $x \in F_{y}$, the germ of $\gamma$ at $x$ is a $\mathbf{Z} / 2$-versal deformation of $\left(F_{y}, x\right)$. Thus $\mathscr{G}=\{(A, \Theta): \gamma$ is a $\mathbf{Z} / 2$-versal deformation of its fibers $\}$; cf. (2.25).

(3.12) Proposition. $\mathscr{G}$ is Zariski-open in $\mathscr{A}_{3}$.

Proof. We first replace $\mathscr{A}_{3}$ by a finite cover which carries a family of theta divisors. There exists a variety $\tilde{\mathscr{A}}_{3}$ and an algebraic family $(\mathbf{A}, \mathbf{\theta}) \rightarrow \tilde{\mathscr{A}}_{3}$ of principally polarized abelian varieties such that the classifying map $\tilde{\mathscr{A}}_{3} \rightarrow$ $\mathscr{A}_{3}$ is a finite surjective morphism. (We can take $\tilde{\mathscr{A}}_{3}$ to be a moduli space parametrizing abelian varieties together with a level $n$ structure; cf. [18, 7.9, p. 139].) Let $\mathscr{A}_{3}^{\prime}=\left\{s \in \tilde{\mathscr{A}}_{3}: \Theta_{s}\right.$ is smooth $\}$, and let $\mathscr{G}^{\prime}=\left\{s \in \tilde{\mathscr{A}}_{3}: \boldsymbol{\Theta}_{s}\right.$ is Gauss-stable $\}$. It suffices to show that $\mathscr{G}^{\prime}$ is open in $\mathscr{A}_{3}^{\prime}$.

Over $\mathscr{A}_{3}^{\prime}$ there is a Gauss map $\gamma$ for the family $(\mathbf{A}, \mathbf{\theta})$ :

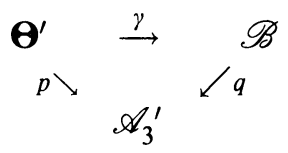

Here $\mathscr{B}$ is the Zariski fiber bundle with fibers $B_{s}=\mathbf{P} T_{0}^{*}\left(A_{s}\right)$, and $\gamma_{s}$ is the Gauss map of $\Theta_{s}$. Let $\mathscr{F}$ be the cokernel sheaf of the Kodaira-Spencer map

$$
\mathscr{T}_{\mathscr{B} / \mathscr{A}_{3}^{\prime}} \rightarrow\left(\gamma_{*}\left(\mathscr{T}_{\mathbf{\Theta}^{\prime} / \mathscr{B}}^{1}\right)\right)^{+}
$$

defined in (3.5). The support of $\mathscr{F}$ is the locus of points $y \in \mathscr{B}$ for which the Gauss map $\gamma_{s}, s=q(y)$, is not a $\mathbf{Z} / 2$-versal deformation of the fiber $\gamma_{s}^{-1}(y)=F_{y}$. Since $\mathscr{F}$ is a coherent algebraic sheaf, Supp $\mathscr{F}$ is Zariski-closed in $\mathscr{B}$, and since $q$ is proper, $q(\operatorname{Supp} \mathscr{F})=\mathscr{A}_{3}^{\prime}-\mathscr{G}^{\prime}$ is Zariski-closed in $\mathscr{A}_{3}^{\prime}$.

Now we turn to the proof that $\mathscr{G}$ is nonempty.

(3.13) Proposition. There exists a family $p: \mathscr{X} \rightarrow \Delta$ over the disc, together with a flat morphism $f: \mathscr{X} \rightarrow \mathbf{P}^{2 *} \times \Delta$ and an involution $l: \mathscr{X} \rightarrow \mathscr{X}$, with the following properties:

(a) $\mathscr{X}$ is nonsingular and $p$ is a proper, flat map. For $t \neq 0$ the fibers $X_{t}$ are smooth genus 3 theta divisors $\Theta_{t}$, and the fiber $X_{0}$ is the union of the Nash blow up $\widehat{\Theta}_{0}$ of a hyperelliptic theta divisor and a smooth quadric surface $Q$, with $\widehat{\Theta}_{0}$ and $Q$ meeting transversely along the exceptional divisor $E$ in $\widehat{\Theta}_{0}$.

(b) $p=q \circ f$, where $q: \mathbf{P}^{2 *} \times \Delta \rightarrow \Delta$ is projection to the second factor. For $t \neq 0$, the induced map $f_{t}: X_{t} \rightarrow \mathbf{P}^{2 *}$ is the Gauss map of $\boldsymbol{\Theta}_{t}$. The map $f_{0}: X_{0} \rightarrow \mathbf{P}^{2 *}$ restricts on $\widehat{\Theta}_{0}$ to the Nash blowup of the Gauss map of $\Theta_{0}$, and $f_{0}$ restricts on $Q$ to a double cover branched along $E$.

(c) $p \circ l=p$ and $f \circ l=f$. For $t \neq 0$ the induced map $l_{t}: X_{t} \rightarrow X_{t}$ is the natural involution of $\Theta_{t}$. The involution $l_{0}$ restricts on $\widehat{\Theta}_{0}$ to the Nash extension of the natural involution of $\Theta_{0}$, and $l_{0}$ restricts on $Q$ to a nontrivial involution with fixed point set $E$.

Proof. Let $(\Delta, 0)$ be the germ of a smooth arc in Siegel space $\mathscr{H}_{3}$ (cf. [1, 20, 22]) through a hyperelliptic Jacobian $\left(J_{0}, \Theta_{0}\right)$, and let $\boldsymbol{\theta}$ be the total space of the induced one-parameter family of theta divisors. We choose $\Delta$ transverse to the hyperelliptic locus; then $\boldsymbol{\theta}$ is nonsingular. (This follows from the fact that 
the singular point of a hyperelliptic genus 3 theta divisor is an ordinary double point.) Consider the (rational) relative Gauss map $\gamma: \boldsymbol{\theta} \rightarrow \mathbf{P}^{2 *} \times \Delta$ of the family, which induces, for each $t \in \Delta$, the Gauss map $\gamma_{t}$ on the theta divisor $\boldsymbol{\Theta}_{t}$. Let $\hat{\gamma}: \widehat{\boldsymbol{\theta}} \rightarrow \mathbf{P}^{2 *} \times \Delta$ be the extension of $\gamma$ to a morphism on the relative Nash blowup $\widehat{\boldsymbol{\theta}}$, which is isomorphic to the blowup of $\boldsymbol{\theta}$ at the double point $\xi$ of $\Theta_{0}$ (cf. [23]). The central fiber of $\widehat{\boldsymbol{\theta}}$ is $\widehat{\Theta}_{0}+2 \mathscr{E}$, with $\mathscr{E}=\mathbf{P} T_{\xi}(\mathbf{\theta})$. The two divisors $\widehat{\boldsymbol{\Theta}}_{0}$ and $\mathscr{E}$ of $\widehat{\boldsymbol{\Theta}}$ meet transversely along a smooth rational curve. In $\widehat{\Theta}_{0}$ this curve is the $g_{2}^{1}$ curve $E$, and in $\mathscr{E}$ this curve is a conic. The induced map $\hat{\gamma}_{0}: \widehat{\boldsymbol{\Theta}}_{0}+\mathscr{E} \rightarrow \mathbf{P}^{2 *}$ on the reduced central fiber restricts on $\widehat{\boldsymbol{\Theta}}_{0}$ to the Nash resolution of the Gauss map $\gamma_{0}: \Theta_{0} \rightarrow \mathbf{P}^{2}$, and the induced map $\mathscr{E} \rightarrow \mathbf{P}^{2 *}$ is an isomorphism. The involution $l$ on $\boldsymbol{\theta}$ lifts to an involution $\hat{\imath}$ of $\widehat{\boldsymbol{\theta}}$ which is the identity on $\mathscr{E}$.

We obtain the family $\mathscr{X}$ from $\widehat{\boldsymbol{\Theta}}$ by base change and normalization. First we make the base change $s \rightarrow s^{2}=t$ to the $s$-disc $\Delta^{\prime}$, and consider the induced family $\widehat{\boldsymbol{\Theta}}^{\prime}$ with morphism $\hat{\gamma}^{\prime}: \widehat{\boldsymbol{\theta}}^{\prime} \rightarrow \mathbf{P}^{2 *} \times \Delta^{\prime}$ and involution $\hat{\imath}^{\prime}(x, s)=$ $(\hat{\imath}(x), s)$. The total space $\hat{\boldsymbol{\Theta}}^{\prime}$ is a double cover of $\widehat{\boldsymbol{\Theta}}$ branched along $\widehat{\boldsymbol{\Theta}}_{0}+2 \mathscr{E}$. Let $\widetilde{\boldsymbol{\theta}} \rightarrow \widehat{\boldsymbol{\Theta}}^{\prime}$ be the normalization of $\widehat{\boldsymbol{\Theta}}^{\prime}$. Then $\widetilde{\boldsymbol{\theta}}$ is nonsingular, and $\widetilde{\boldsymbol{\theta}} \rightarrow \Delta$ is the desired family $\mathscr{X} \rightarrow \Delta$. The map $f$ is the pullback to $\widetilde{\boldsymbol{\theta}}$ of $\hat{\gamma}$.

To see that the induced involution $\tilde{l}$ of $\tilde{\boldsymbol{\theta}}$ acts nontrivially on $Q$, the preimage of $\mathscr{E}$, we must check that $\hat{l}^{\prime}$ interchanges the two branches of $\widehat{\boldsymbol{\Theta}}^{\prime}$ along $\mathscr{E}$. Now $\hat{l}^{\prime}$ is the restriction of the involution $\kappa(x, s)=(\hat{\imath}(x), s)$ of $\widehat{\boldsymbol{\theta}} \times \Delta^{\prime}$. For a point $x$ of $\mathscr{E}$ not in $\widehat{\boldsymbol{\Theta}}_{0}$, the derivative $\kappa_{*}$ acts on the normal plane $N_{(x, 0)}$ to $\mathscr{E} \times\{0\}$ in $\widehat{\boldsymbol{\Theta}} \times \Delta^{\prime}$, preserving the normal cone $C_{x}$ to $\mathscr{E}$ in $\widehat{\boldsymbol{\Theta}}^{\prime}$. It suffices to show $\kappa_{*}$ interchanges the two lines of $C_{x}$. If not, the two lines would be eigenspaces of $\kappa_{*}$, and their eigenvalues $( \pm 1)$ would be equal, since the two branches of $\widehat{\boldsymbol{\Theta}}^{\prime}$ can be interchanged by monodromy. This would contradict that the fixed point set of $\kappa_{*}$ has codimension one. The remaining properties of the family $\mathscr{X} \rightarrow \Delta$ follow immediately.

Now we compute the space of first-order $Z / 2$-deformations of a fiber of the map $f_{0}: X_{0} \rightarrow \mathbf{P}^{2 *}$ constructed in (3.13), and compare with the deformations of the corresponding fiber of the Nash Gauss map. We have $X_{0}=\widehat{\Theta}_{0} \cup Q$ and $\hat{\gamma}_{0}=f_{0} \mid \widehat{\Theta}_{0}$; set $\mu=f_{0} \mid Q$. Locally in $\mathbf{C}^{3}$, with coordinates $(u, v, w)$, we realize $X_{0}$ as $u w=0$, with $\widehat{\Theta}_{0}$ given by $w=0$ and $Q$ given by $u=0$. Choosing local coordinates $(a, b)$ on $\mathrm{P}^{2 *}$, we may assume $\mu(v, w)=\left(w^{2}, v\right)$, and so $\hat{\gamma}_{0}(u, v)=(u g(u, v), v+u h(u, v))$ for some analytic functions $g$ and $h$. (Since $\hat{\gamma}_{0}$ has rank 1 along $E=\widehat{\Theta}_{0} \cap Q$ (by $\mathbf{Z} / 2$-symmetry), $g=$ $u k(u, v)$ for some analytic function $k$.) Then $f$ is given locally on $X_{0}$ by $(a, b)=\left(u g+w^{2}, v+u h\right)$. (The components of $f$ are unique up to addition of functions divisible by $u w$.$) The ideal in A=\mathscr{O}_{\mathbf{C}^{3}, 0}$ of the local schemetheoretic fiber $(F, x)=\left(f^{-1}(0), 0\right)$ is $I=\langle u w, a, b\rangle$. We compute that $T_{(F, x)}^{1}=A^{3} / J$, where

$$
\begin{aligned}
J= & \left\langle u w, u g+w^{2}, v+u h\right\rangle A^{3}+A\left(w, g+u g_{u}, h+u h_{u}\right) \\
& +A\left(0, u g_{v}, 1+u h_{v}\right)+A(u, 2 w, 0) .
\end{aligned}
$$


Let $\left(F^{\prime}, x\right)$ be the local scheme-theoretic fiber $\left(\left(\hat{\gamma}_{0}\right)^{-1}(0), 0\right)$. The ideal in $A$ of $\left(F^{\prime}, x\right)$ is $I^{\prime}=\langle w, a, b\rangle$, so $T_{\left(F^{\prime}, x\right)}^{1}=A^{3} / J^{\prime}$, where

$$
\begin{aligned}
J= & \left\langle w, u g+w^{2}, v+u h\right\rangle A^{3}+A\left(0, g+u g_{u}, h+u h_{u}\right) \\
& +A\left(0, u g_{v}, 1+u h_{v}\right)+A(1,2 w, 0) .
\end{aligned}
$$

(3.14) Lemma. $\operatorname{dim}\left(T_{(F, x)}^{1}\right)^{+}=\operatorname{dim}\left(T_{\left(F^{\prime}, x\right)}^{1}\right)^{+}+1$.

Proof. Clearly $J \subset J^{\prime}$ and $(1,0,0) \in J^{\prime}$. Let $\sigma$ be the image of $(1,0,0)$ in $V=J^{\prime} / J$. One checks that $\sigma$ generates $V$ as an $A$-module, and that $u^{2}$, $v+h(0,0) u, w^{2}$ annihilate $\sigma$. It follows that $\sigma, u \sigma$, and $w \sigma$ span $V$ as a complex vector space, and hence $\sigma$ spans $V^{+}$.

(3.15) Proposition. $\mathscr{G}$ is nonempty.

Proof. We apply (3.10) to the family $f: \mathscr{X} \rightarrow \mathscr{Y}=\mathbf{P}^{2 *} \times \Delta$ of (3.13). We must check, for all $y \in Y_{0}=\mathbf{P}^{2 *} \times\{0\}$, that the Kodaira-Spencer map $\varphi_{y}: T_{y}\left(Y_{0}\right) \rightarrow$ $\left(T_{F_{y}}^{1}\right)^{+}$is surjective if $X_{0}$ is smooth at all points of $F_{y}$, and that $\operatorname{dim}\left(\operatorname{coker}\left(\varphi_{y}\right)\right)$ $\leq 1$ if $X_{0}$ is singular at some point of $F_{y}$. The singular locus of $X_{0}=\widehat{\Theta}_{0} \cup Q$ is $E=\widehat{\Theta}_{0} \cap Q$. Recall that $f \mid Q$ is a double cover branched along $E$, and that $f \mid \widehat{\Theta}_{0}=\hat{\gamma}_{0}$, the Nash Gauss map. The fibers $F_{y}=f^{-1}(y)$ have length 6 , and the fibers $F_{y}^{\prime}=\left(\hat{\gamma}_{0}\right)^{-1}(y)$ have length $4 . \quad F_{y}$ has at most one $l$-orbit of singular points, which is also the unique $l$-orbit of singular points of $F_{y}^{\prime}$ (2.26). For $x$ in this orbit, $\left(T_{F}^{1}\right)^{+}=\left(T_{(F, x)}^{1}\right)^{(\mathbf{Z} / 2)_{x}}$ and $\left(T_{F^{\prime}}^{1}\right)^{+}=\left(T_{\left(F^{\prime}, x\right)}^{1}\right)^{(\mathbf{Z} / 2)_{x}}$. We know from $(2.1 \mathrm{~b}$ ) (which can be proved by direct computation based on (2.26)) that $\varphi_{y}: T_{y}\left(\mathbf{P}^{2 *}\right) \rightarrow\left(T_{F^{\prime}}^{1}\right)^{+}$is surjective. Thus if $F_{y}$ does meet $E$, then $\operatorname{dim}\left(\operatorname{coker}\left(\varphi_{y}\right)\right) \leq 1$ by $(3.14)$. If $F_{y}$ does not meet $E$, then $\left(T_{F^{\prime}}^{1}\right)^{+}=\left(T_{F}^{1}\right)^{+}$, and so $\varphi_{y}: T_{y}\left(\mathbf{P}^{2 *}\right) \rightarrow\left(T_{F}^{1}\right)^{+}$is surjective.

This completes the dynamic proof of Theorem (3.1).

\section{REFERENCES}

1. E. Arbarello, M. Cornalba, P. Griffiths, and J. Harris, Geometry of algebraic curves, vol. I, Springer-Verlag, New York, 1985.

2. V. I. Arnold, S. M. Gusein-Zade, and A. N. Varchenko, Singularities of differentiable maps. I, Birkhäuser, Boston, Mass., 1985.

3. E. Bierstone, Local properties of smooth maps equivariant with respect to finite group actions, J. Differential Geom. 10 (1975), 523-540.

4. __ Generic equivariant maps, Real and Complex Singularities, (P. Holm, ed.), Sijthoff and Noordhoff, Alphen aan den Rijn, 1977, pp. 127-161.

5. _ The structure of orbit spaces and the singularities of equivariant mappings, Monografias de Matemática, no. 35, IMPA, Rio de Janiero, 1980.

6. H. Cartan, Quotient d'un espace analytique par un groupe d'automorphismes, Algebraic Geometry and Topology, Princeton Univ. Press, Princeton, N. J., 1957, pp. 90-102.

7. A. Douady and J.-L. Verdier, Séminaire de géométrie analytique, École Norm. Sup. 19711972, Astérisque 16 (1974).

8. R. Friedman and R. Smith, Degenerations of Prym varieties and intersections of three quadrics, Invent. Math. 85 (1986), 615-635.

9. P. Griffiths and J. Harris, Principles of algebraic geometry, Wiley, New York, 1978.

10. R. Hartshorne, Algebraic geometry, Springer-Verlag, New York, 1977. 
11. L. Illusie, Complexe cotangent et déformations. I, Lecture Notes in Math., vol. 239, SpringerVerlag, New York, 1971.

12. A. Kas and M. Schlessinger, On the versal deformation of a complex space with an isolated singularity, Math. Ann. 196 (1972), 23-29.

13. S. Lichtenbaum and M. Schlessinger, The cotangent complex of a morphism, Trans. Amer. Math. Soc. 128 (1967), 41-70.

14. E. J. N. Looijenga, Isolated singular points on complete intersections, London Math. Soc. Lecture Notes 77, Cambridge Univ. Press, 1984.

15. C. McCrory and T. Shifrin, Cusps of the projective Gauss map, J. Differential Geom. 19 (1984), 257-276.

16. C. McCrory, T. Shifrin, and R. Varley, The Gauss map of a generic hypersurface in $\mathbf{P}^{4}, \mathbf{J}$. Differential Geom. 30 (1989), 689-759.

17. C. McCrory, T. Shifrin, R. Varley, and M. Adams, Symmetric Lagrangian singularities and Gauss maps of theta divisors, Lectures Notes in Math., vol. 1462, Springer-Verlag, New York, 1991, pp. 1-26.

18. D. Mumford and J. Fogarty, Geometric invariant theory, Springer-Verlag, Berlin, 1982.

19. V. Poénaru, Singularités $C^{\infty}$ en présence de symétrie, Lecture Notes in Math., vol. 510, Springer-Verlag, Berlin, 1976.

20. H. E. Rauch, A transcendental view of the space of algebraic Riemann surfaces, Bull. Amer. Math. Soc. 71 (1965), 1-39.

21. D. Rim, Equivariant G-structure on versal deformations, Trans. Amer. Math. Soc. 257 (1980), 217-226.

22. R. Smith and R. Varley, Components of the locus of singular theta divisors of genus 5 , Lecture Notes in Math., vol. 1124, Springer-Verlag, New York, 1985, pp. 338-416.

23. B. Teissier, Variétés polaires, II: multiplicités polaires, sections planes, et conditions de Whitney, Lecture Notes in Math., vol. 961, Springer-Verlag, New York, 1981, pp. 314-491.

24. A. M. Vermuelen, Weierstrass points of weight two on curves of genus three, Dissertation, University of Amsterdam, 1983.

Department of Mathematics, University of Georgia, Athens, Georgia 30602

E-mail address: clint@joe.math.uga.edu

E-mail address: shifrin@joe.math.uga.edu

E-mail address: robert@joe.math.uga.edu 